$7-1-2008$

\title{
Radiation Torque Exerted on a Spheroid: Analytical Solution
}

Feng Xu

James A. Lock

Cleveland State University, j.lock@csuohio.edu

Gérard Gouesbet

Cameron Tropea

Follow this and additional works at: https://engagedscholarship.csuohio.edu/sciphysics_facpub

Part of the Physics Commons

How does access to this work benefit you? Let us know!

Publisher's Statement

Copyright 2008 American Physical Society. Available on publisher's site at http://pra.aps.org/ abstract/PRA/v78/i1/e013843.

\section{Original Citation}

Xu, Feng, James A. Lock, Gerard Gouesbet, and Cameron Tropea. "Radiation Torque Exerted on a Spheroid: Analytical Solution." Physical Review A 78 (2008): 13843.

\section{Repository Citation}

Xu, Feng; Lock, James A.; Gouesbet, Gérard; and Tropea, Cameron, "Radiation Torque Exerted on a Spheroid: Analytical Solution" (2008). Physics Faculty Publications. 51.

https://engagedscholarship.csuohio.edu/sciphysics_facpub/51

This Article is brought to you for free and open access by the Physics Department at EngagedScholarship@CSU. It has been accepted for inclusion in Physics Faculty Publications by an authorized administrator of EngagedScholarship@CSU. For more information, please contact library.es@csuohio.edu. 


\title{
Radiation torque exerted on a spheroid: Analytical solution
}

\author{
Feng $\mathrm{Xu}^{*}$ \\ Fachgebiet Strömungslehre und Aerodynamik, Technische Universität Darmstadt, Petersenstrass 30, 64287 Darmstadt, Germany \\ and Institute of Particle and Two-Phase Flow Measurement, University of Shanghai for Science and Technology, Shanghai 200093, China \\ James A. Lock \\ Department of Physics, Cleveland State University, Cleveland, Ohio 44115, USA \\ Gérard Gouesbet \\ LESP, UMR 6614/CORIA CNRS, Université et INSA de Rouen, Site du Madrillet, Avenue de l'Université, BP12 76801 \\ Saint Etienne du Rouvray, France \\ Cameron Tropea \\ Fachgebiet Strömungslehre und Aerodynamik, Technische Universität Darmstadt, Petersenstrass 30, 64287 Darmstadt, Germany
}

(Received 12 March 2008; published 29 July 2008)

\begin{abstract}
As a companion work to our previous study [F. Xu, et al., Phys. Rev. E. 75, 026613 (2007)] on radiation force prediction for a homogeneous spheroid, we provide in this paper the analytical solution to the radiation torque exerted by an arbitrarily shaped beam on a spheroid, which can be prolate or oblate, transparent or absorbing. Calculations based upon this theoretical development are performed for both linearly and circularly polarized incident beams, and the results are compared to those of a sphere. Stable orientations of spheroids inside a linearly and a circularly polarized Gaussian beam are predicted. We analyze two physical mechanisms, the polarization torque and the reaction force torque, which do not exist or have no contribution to the torque on a sphere but cause rotation of a spheroid. As verification, the dipole method is also developed for the torque calculation for spheroids of size much less than the wavelength, and geometrical optics is developed to qualitatively analyze the torque exerted on spheroids of large size.
\end{abstract}

DOI: 10.1103/PhysRevA.78.013843

PACS number(s): 42.25.Fx, 87.80.Cc

\section{INTRODUCTION}

Transfer of momentum and angular momentum from a laser beam to a particle are known to produce radiation forces and torques, which can be employed to manipulate and characterize microscopic objects [1-8]. For example, viruses, bacteria, organelles, and even DNA strands can be trapped by a highly focused laser beam inducing forces in piconewton range [6]. The orientation of nonspherical particles can be controlled through the torque exerted by laser beams carrying spin angular momentum [7]. By using two counterpropagating laser beams stretching forces can be induced to measure the mechanical rigidity of a cell [8].

Scientific design and optimization of optical instruments implementing these measurements requires the relevant development of scattering theory describing the interaction between laser beams and particles. Though rigorous electromagnetic scattering theory with analytical solution to the radiation force and torque exerted on a homogeneous and radially inhomogeneous sphere have been well established [9-15], much less work has been reported for nonspherical particles, except for the case of a cylinder with circular cross section [16]. To deal with the complex particle shapes to which analytical solution is hard to obtain or does not exist, the numerical approaches, e.g., the surface-based $T$-matrix method $[17,18]$ and volume-based finite-difference time-

*f3_xu@yahoo.com domain method [19] have been developed. To avoid the mathematical difficulties in establishing the rigorous theory, some approximate approaches, e.g., the geometrical optics approximation [20-23] and Rayleigh theory [24,25] become alternatives when extreme precision is not of primary importance. For example, to evaluate the force and torque exerted on many regularly shaped particles of large size, e.g., spheroid, cylinder, ring-shaped particle, etc., geometrical optics can be employed [20-23]. In the other limit, when the particle size is considerably smaller than the wavelength, Rayleigh theory provides an efficient choice [24,25]. For some regularly shaped particles, e.g., spheres, cylinders, spheroids, etc., these two limiting cases can be unified by the rigorous theory called "generalized Lorenz-Mie theory" (GLMT) [26]. Within the framework of GLMT, once the beam is expanded in relevant coordinates mirroring the geometry of the particle, analytical solutions to all physical quantities, e.g., scattered intensities, scattering and extinction cross sections, and radiation forces and torques are available.

Obviously, the extension of GLMT from sphere to some nonspherical particles is significant since most particles existing in nature or generated in industrial processes are not exactly spherical. Even for some soft spherical particles, e.g., droplets and biological cells, the larger they are, the easier it is to deform them, due to the decreasing efficiency of surface tension to maintain their sphericity. Actually, when these particles are only slightly deformed from spherical shape they might be well modeled by a spheroid, e.g., optically or acoustically levitated droplets, atomized droplets, etc. For their characterization by optical means, a rigorous theory 
with analytical solution describing their scattering characteristics is of great importance.

In the past decade, GLMT for a spheroid has been developed stepwise. Analytical solutions to scattering amplitudes, extinction, and scattering cross sections, as well as radiation forces, were successively found $[27,28]$. The present contribution adds the torque to this list.

Before introducing our work, we briefly look back at the physical mechanisms of the torque exerted on a sphere by a laser beam. First, we consider the simplest system of a sphere illuminated by a plane wave. To generate torque on the particle, Marston and Crichton found the plane wave must be circularly polarized and the sphere must be absorbing [9]. A circularly polarized plane wave carries spin angular momentum [29]. When it is incident on an absorbing particle, part of circular polarization angular momentum is lost by the incident beam and transported to the sphere. We call this the spin-absorption mechanism, which also occurs for the case of a sphere illuminated by an on-axis shaped beam. Next, we consider the system of a sphere illuminated by an off-axis shaped beam. As found by Chang and Lee [12] and Barton [14], a torque can be induced by a linearly polarized shaped beam. We call this the orbit-absorption mechanism since an off-axis beam has orbital angular momentum with respect to the sphere's axis and this orbital angular momentum is transferred to the rotation of the sphere through absorption. The third circumstance is that the sphere is illuminated by an off-axis circularly polarized beam. The torque induced in this case was also predicted by Chang and Lee [12] and Barton [14]. It can be attributed to both the spinabsorption and orbit-absorption mechanisms. All these mechanisms explaining the torque exerted on a sphere also occur for a spheroid. The majority of the results presented in this paper will be for the geometries where the torque on a sphere is zero. Two types of torques which do not occur for spheres, i.e., the polarization torque and reaction force torque, are identified and analyzed.

\section{THEORY}

According to the conservation law of angular momentum, the radiation torque exerted on a particle of arbitrary shape is equal to the average rate at which the angular momentum is conveyed to it. Mathematically, it can be expressed as a surface integral of the dot product of the outward normal unit vector $\hat{n}$ and the pseudotensor $\overleftrightarrow{A} \times \mathbf{r}$ over a surface enclosing the particle designated by $S[30,31]$,

$$
\langle\mathbf{T}\rangle=-\int_{S} \hat{n} \cdot\langle\overleftrightarrow{A}\rangle \times \mathbf{r} d S
$$

where the symbol \langle\rangle denotes time average and $\overleftrightarrow{A}$ is the Maxwell stress tensor expressed by

$$
\overleftrightarrow{A}=\varepsilon \mathbf{E E}^{*}+\mu \mathbf{H} \mathbf{H}^{*}-\frac{1}{2}\left(\varepsilon|\mathbf{E}|^{2}+\mu|\mathbf{H}|^{2}\right) \stackrel{\leftrightarrow}{I}
$$

where $\mathbf{E}$ and $\mathbf{H}$ are the total electric and magnetic fields outside the particle, $\stackrel{\leftrightarrow}{I}$ is the unit tensor, $\varepsilon$ and $\mu$ denote the

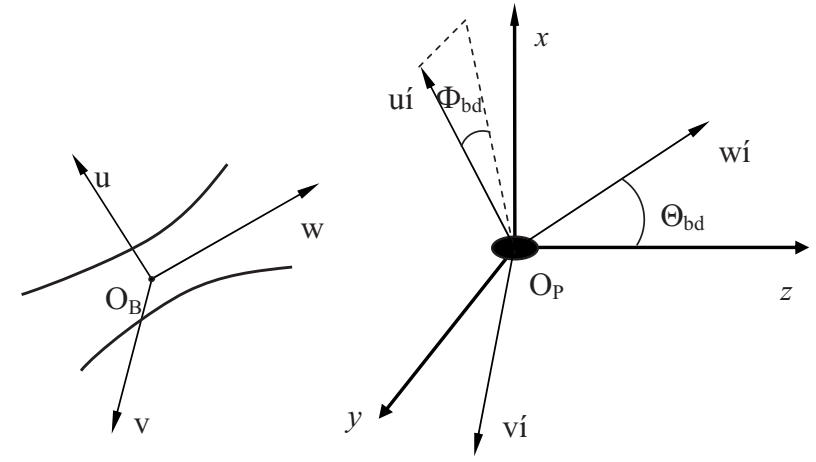

FIG. 1. Coordinate systems: $O_{\mathrm{B}}-u v w$ is attached to the incident shaped beam and $O_{\mathrm{P}^{-}} x y z$ is attached to the spheroid.

permittivity and permeability of the medium, respectively, and the asterisk indicates the complex conjugate.

\section{A. Description of the electromagnetic fields}

We consider a monochromatic arbitrarily oriented shaped beam whose electric field is linearly polarized in the $u$ direction at the waist in its own Cartesian coordinates $O_{B}-u v w$. The time-dependent part of its electromagnetic fields is $\exp (-i \omega t)$, with $\omega$ being the angular frequency. Such a beam is incident on a spheroid of semimajor axis length $a$ and semiminor axis length $b$. As indicated by Fig. 1, through coordinate translation the beam center, $O_{\mathrm{B}}$ can be moved to the particle center $O_{\mathrm{P}}$ to generate the system $O_{\mathrm{P}}-u^{\prime} v^{\prime} w^{\prime}$. Taking the axis of symmetry of the spheroid as the $z$ axis, we set the $x$ axis of the Cartesian coordinates of the particle to lie in the plane formed by the $w^{\prime}$ and $z$ axes. This determines the particle coordinate system $O_{\mathrm{P}^{-}} x y z$. Then the two coordinate systems can be related by two beam angles. One is the incident angle $\Theta_{\text {bd }}$ indicating the beam propagation direction in $O_{\mathrm{P}}-x y z$, and the other is the polarization angle $\Phi_{\mathrm{bd}}$ indicating the polarization direction of the beam relative to the $O_{\mathrm{P}}-x z$ plane. Note that the signs of $\Theta_{\mathrm{bd}}$ and $\Phi_{\mathrm{bd}}$ are the same as those of cross products of unit vectors, $\boldsymbol{e}_{z} \times \boldsymbol{e}_{w}$ and $\boldsymbol{e}_{u_{\mathrm{P}}} \times \boldsymbol{e}_{u}$ respectively, with $u_{\mathrm{P}}$ denoting the projection of $u$ axis in the $O_{\mathrm{P}^{-}} x z$ plane. These two angles, in conjunction with the coordinates of beam center $\left(x_{0}, y_{0}, z_{0}\right)$ in $O_{\mathrm{P}^{-}} x y z$ are used to evaluate the spheroidal beam shape coefficients (or more briefly, "spheroidal BSCs"), $G_{n, T E}^{m}$ and $G_{n, T M}^{m}$, for an arbitrarily shaped beam [32].

When the spheroidal BSCs are obtained, the incident fields $\left(\mathbf{E}^{(\mathrm{i})}, \mathbf{H}^{(\mathrm{i})}\right)$ can be expanded in terms of spheroidal vector functions $\left(\mathbf{M}_{m n}, \mathbf{N}_{m n}\right)$ in the spheroidal coordinates $(\xi, \eta, \phi)$

$$
\begin{aligned}
\mathbf{E}^{(i)}= & \sum_{m=-\infty}^{\infty} \sum_{n=|m|, n \neq 0}^{\infty} i^{n+1}\left[i G_{n, T E}^{m} \mathbf{M}_{m n}^{(i)}\left(C_{\mathrm{I}} ; \xi, \eta, \phi\right)\right. \\
& \left.+G_{n, T M}^{m} \mathbf{N}_{m n}^{(i)}\left(C_{\mathrm{I}} ; \xi, \eta, \phi\right)\right], \\
\mathbf{H}^{(i)}=- & \frac{i k_{\mathrm{I}}}{\omega \mu_{\mathrm{I}}} \sum_{m=-\infty}^{\infty} \sum_{n=|m|, n \neq 0}^{\infty} i^{n+1}\left[G_{n, T M}^{m} \mathbf{M}_{m n}^{(i)}\left(C_{\mathrm{I}} ; \xi, \eta, \phi\right)\right. \\
+ & \left.i G_{n, T E}^{m} \mathbf{N}_{m n}^{(i)}\left(C_{\mathrm{I}} ; \xi, \eta, \phi\right)\right],
\end{aligned}
$$

where $k_{\mathrm{I}}$ is the wave number of the surrounding medium 
denoted by the subscript $I$ and assumed to be linear, isotropic, and nonmagnetic $\left(\mu_{\mathrm{I}}=1\right)$, and $C$ is the size parameter calculated by multiplying the wave number $k_{\mathrm{I}}$ by the semifocal length of the spheroid $f$, namely $C_{\mathrm{I}}=k_{\mathrm{I}} f$ $=k_{\mathrm{I}} a\left[(a / b)^{2}-1\right]^{1 / 2}$. Likewise, the scattered fields $\left(\mathbf{E}^{(\mathrm{s})}, \mathbf{H}^{(\mathrm{s})}\right)$ can be expressed in terms of vector wave functions in the following way:

$$
\begin{aligned}
\mathbf{E}^{(s)}= & \sum_{m=-\infty}^{\infty} \sum_{n=|m|, n \neq 0}^{\infty} i^{n+1}\left[i B_{n}^{m} \mathbf{M}_{m n}^{(s)}\left(C_{\mathrm{I}} ; \xi, \eta, \phi\right)\right. \\
& \left.+A_{n}^{m} \mathbf{N}_{m n}^{(s)}\left(C_{\mathrm{I}} ; \xi, \eta, \phi\right)\right], \\
\mathbf{H}^{(s)}=- & \frac{i k_{\mathrm{I}}}{\omega \mu_{\mathrm{I}}} \sum_{m=-\infty}^{\infty} \sum_{n=|m|, n \neq 0}^{\infty} i^{n+1}\left[A_{n}^{m} \mathbf{M}_{m n}^{(s)}\left(C_{\mathrm{I}} ; \xi, \eta, \phi\right)\right. \\
& \left.+i B_{n}^{m} \mathbf{N}_{m n}^{(s)}\left(C_{\mathrm{I}} ; \xi, \eta, \phi\right)\right],
\end{aligned}
$$

where the scattering coefficients, $A_{n}^{m}$ and $B_{n}^{m}$, are determined via solving the equations established from the boundary conditions ensuring the continuity of tangential components of electric and magnetic vectors across the surface of a spheroid [27]. A superposition of the incident and scattered field gives the total external field for the torque calculation.

In this paper we only present the formulas for a prolate spheroid since all the equations and expressions pertaining to the prolate spheroidal coordinates can be converted to their counterparts in the oblate system through replacing the size parameter $C_{\mathrm{I}}$ by $-i C_{\mathrm{I}}$ and the spheroidal radial coordinate $\xi$ by $i \xi$.

\section{B. Analytical solution to the radiation torque}

To perform the integral indicated by Eq. (1), an arbitrary Gaussian surface surrounding the spheroid can be used. For the sake of mathematical convenience, the surface characterized by an infinite spheroidal radial coordinate $\xi$ is chosen. In this limit this surface is essentially spherical so that we have $f \xi \rightarrow r$ and $\eta \rightarrow \cos \theta$. Then Eq. (1) becomes

$$
\begin{aligned}
\langle\mathbf{T}\rangle= & -\frac{1}{2} \int_{0}^{2 \pi} \int_{0}^{\pi} \operatorname{Re}\left[\left(\varepsilon_{\mathrm{I}} E_{r} E_{\varphi}^{*}+\mu_{\mathrm{I}} H_{r} H_{\varphi}^{*}\right) \vec{e}_{\theta}\right. \\
& \left.-\left(\varepsilon_{\mathrm{I}} E_{r} E_{\theta}^{*}+\mu_{\mathrm{I}} H_{r} H_{\theta}^{*}\right) \vec{e}_{\varphi}\right] r^{3} \sin \theta d \theta d \varphi,
\end{aligned}
$$

where $E=E^{i}+E^{s}$ and $H=H^{i}+H^{s}$. Utilizing the projections of the unit vectors $\vec{e}_{\theta}$ and $\vec{e}_{\varphi}$, we can decompose the torque into the following three components about the $x, y$, and $z$ axes, respectively,

$$
\begin{aligned}
T_{x}= & -\frac{1}{2} \int_{0}^{2 \pi} \int_{0}^{\pi} \operatorname{Re}\left\{\left[\varepsilon_{\mathrm{I}}\left(E_{r}^{s} E_{\varphi}^{s *}+E_{r}^{s} E_{\varphi}^{i *}+E_{r}^{i} E_{\varphi}^{s *}+E_{r}^{i} E_{\varphi}^{i *}\right)\right.\right. \\
& \left.+\mu_{\mathrm{I}}\left(H_{r}^{s} H_{\varphi}^{s *}+H_{r}^{s} H_{\varphi}^{i *}+H_{r}^{i} H_{\varphi}^{s *}+H_{r}^{i} H_{\varphi}^{i *}\right)\right] \cos \theta \cos \varphi \\
& +\left[\varepsilon_{\mathrm{I}}\left(E_{r}^{s} E_{\theta}^{s *}+E_{r}^{i} E_{\theta}^{s *}+E_{r}^{s} E_{\theta}^{i *}+E_{r}^{i} E_{\theta}^{i *}\right)+\mu_{\mathrm{I}}\left(H_{r}^{s} H_{\theta}^{s *}\right.\right. \\
& \left.\left.\left.+H_{r}^{i} H_{\theta}^{s *}+H_{r}^{s} H_{\theta}^{i *}+H_{r}^{i} H_{\theta}^{i *}\right)\right] \sin \varphi\right\} r^{3} \sin \theta d \theta d \varphi,
\end{aligned}
$$

$$
\begin{aligned}
T_{y}= & -\frac{1}{2} \int_{0}^{2 \pi} \int_{0}^{\pi} \operatorname{Re}\left\{\left[\varepsilon_{\mathrm{I}}\left(E_{r}^{s} E_{\varphi}^{s *}+E_{r}^{s} E_{\varphi}^{i *}+E_{r}^{i} E_{\varphi}^{s *}+E_{r}^{i} E_{\varphi}^{i *}\right)\right.\right. \\
& \left.+\mu_{\mathrm{I}}\left(H_{r}^{s} H_{\varphi}^{s *}+H_{r}^{s} H_{\varphi}^{i *}+H_{r}^{i} H_{\varphi}^{s *}+H_{r}^{i} H_{\varphi}^{i *}\right)\right] \cos \theta \sin \varphi \\
& -\left[\varepsilon_{\mathrm{I}}\left(E_{r}^{s} E_{\theta}^{s *}+E_{r}^{i} E_{\theta}^{s *}+E_{r}^{s} E_{\theta}^{i *}+E_{r}^{i} E_{\theta}^{i *}\right)+\mu_{\mathrm{I}}\left(H_{r}^{s} H_{\theta}^{s *}\right.\right. \\
& \left.\left.\left.+H_{r}^{i} H_{\theta}^{s *}+H_{r}^{s} H_{\theta}^{i *}+H_{r}^{i} H_{\theta}^{i *}\right)\right] \cos \varphi\right\} r^{3} \sin \theta d \theta d \varphi \\
T_{z}= & \frac{1}{2} \int_{0}^{2 \pi} \int_{0}^{\pi} \operatorname{Re}\left\{\left[\left(\varepsilon_{\mathrm{I}} E_{r}^{s} E_{\varphi}^{s *}+\mu_{\mathrm{I}} H_{r}^{s} H_{\varphi}^{s *}\right)+\left(\varepsilon_{\mathrm{I}} E_{r}^{i} E_{\varphi}^{i *}\right.\right.\right. \\
& \left.+\mu_{\mathrm{I}} H_{r}^{i} H_{\varphi}^{i *}\right)+\left(\varepsilon_{\mathrm{I}} E_{r}^{s} E_{\varphi}^{i *}+\varepsilon_{\mathrm{I}} E_{r}^{i} E_{\varphi}^{s *}+\mu_{\mathrm{I}} H_{r}^{s} H_{\varphi}^{i *}\right. \\
& \left.\left.\left.+\mu_{\mathrm{I}} H_{r}^{i} H_{\varphi}^{s *}\right)\right] \sin \theta\right\} r^{3} \sin \theta d \theta d \varphi .
\end{aligned}
$$

All the components of electric and magnetic fields contain the spheroidal radial function $R_{m n}$ and the angular function $S_{m n}$ as well as their derivatives [33]. Invoking the orthogonality relationship of the angular wave functions, using the following asymptotic behavior of the radial functions $R_{m n}^{(1)}$ and $R_{m n}^{(3)}$ concerning the incident and scattered fields, respectively:

$$
\begin{gathered}
R_{m n}^{(1)} \rightarrow \frac{1}{2}\left[(-i)^{n+1} \frac{e^{i k r}}{k r}+i^{n+1} \frac{e^{-i k r}}{k r}\right], \\
R_{m n}^{(3)} \rightarrow(-i)^{n+1} \frac{e^{i k r}}{k r},
\end{gathered}
$$

and neglecting the terms of order higher than $1 / r^{2}$, the analytical solution to the radiation torque can be found in series form after a great deal of algebra,

$$
\begin{aligned}
T_{x}+i T_{y}= & \frac{M_{0}}{c} \frac{\pi}{k_{\mathrm{I}}^{3}} \sum_{p=-\infty}^{+\infty} \sum_{n=|p| \neq 0}^{+\infty} \sum_{n^{\prime}=|p-1| \neq 0}^{+\infty}\left(\lambda_{|p| n} J_{n n^{\prime}, 1}^{p} \mp C_{\mathrm{I}}^{2} J_{n n^{\prime}, 2}^{p}\right) \\
& \times\left(A_{n}^{p *} G_{n^{\prime}, T M}^{p-1}+A_{n^{\prime}}^{p-1} G_{n, T M}^{p *}+B_{n}^{p *} G_{n^{\prime}, T E}^{p-1}\right. \\
& \left.+B_{n^{\prime}}^{p-1} G_{n, T E}^{p *}+2 A_{n}^{p *} A_{n^{\prime}}^{p-1}+2 B_{n}^{p *} B_{n^{\prime}}^{p-1}\right) \\
T_{z}= & \frac{2 M_{0}}{c} \frac{\pi}{k_{\mathrm{I}}^{3}} \sum_{p=-\infty}^{+\infty} \sum_{n=|p| \neq 0}^{+\infty} \sum_{n^{\prime}=|p| \neq 0}^{+\infty}\left(\lambda_{|p| n} H_{n n^{\prime}, 1}^{p} \mp C_{\mathrm{I}}^{2} H_{n n^{\prime}, 2}^{p}\right) \\
& \times \operatorname{Re}\left(A_{n^{\prime}}^{p} G_{n, T M}^{p *}+B_{n^{\prime}}^{p} G_{n, T E}^{p *}+A_{n}^{p *} A_{n^{\prime}}^{p}+B_{n}^{p *} B_{n^{\prime}}^{p}\right) .
\end{aligned}
$$

In Eqs. (13) and (14), $\lambda_{|p| n}$ is the separation constant of the differential equations for spheroidal radial and angular functions in spheroidal coordinates [33], $M_{0}$ is the refractive index of the medium, $c$ is the speed of light in vacuum, the minus (plus) sign $\{\mp\}$ corresponds to the prolate-(oblate) spheroid, and $J_{n n^{\prime}, 1}^{p}, J_{n n^{\prime}, 2}^{p}, H_{n n^{\prime}, 1}^{p}$, and $H_{n n^{\prime}, 2}^{p}$ are the orthogonality relationship of the angular functions and are given by 


$$
\begin{aligned}
& J_{n n^{\prime}, 1}^{p}=-\int_{0}^{\pi} S_{|p| n}\left[(p-1) S_{|p-1| n^{\prime}} \cos \theta-\frac{d S_{|p-1| n^{\prime}}}{d \theta} \sin \theta\right] d \theta=0, \quad\left|n-n^{\prime}\right|=\text { odd }, \\
& =2 \sum_{r=2,1}^{\infty} \frac{1}{2|p|+2 r+1} \frac{(2|p|+r+1) !}{(r-1) !} d_{r}^{|p| n} d_{r-1}^{|p-1| n^{\prime}}, \quad\left|n-n^{\prime}\right|=\text { even, } \quad p \leq 0 \text {, } \\
& =-2 \sum_{r=0,1}^{\infty} \frac{1}{2|p|+2 r+1} \frac{(2|p|+r) !}{r !} d_{r}^{|p| n} d_{r+1}^{p-1 \mid n^{\prime}}, \quad\left|n-n^{\prime}\right|=\text { even, } \quad p>0, \\
& J_{n n^{\prime}, 2}^{p}={\overline{\cos ^{2} \theta}}_{n n^{\prime}, 1}^{p}=-\int_{0}^{\pi} S_{|p| n}\left[(p-1) S_{|p-1| n^{\prime}} \cos \theta-\frac{d S_{|p-1| n^{\prime}}}{d \theta} \sin \theta\right] \cos ^{2} \theta d \theta=0, \quad\left|n-n^{\prime}\right|=\text { odd, } \\
& =2\left[\sum_{r=2,1}^{\infty}\left(\frac{2|p|+r}{2|p|+2 r+1}\right)^{2} \frac{r(2|p|+r+1)}{2|p|+2 r-1} \frac{(2|p|+r-1) !}{(r-1) !} d_{r}^{p \mid n} d_{r-1}^{p-1 \mid n^{\prime}}\right. \\
& +\sum_{r=4,3}^{\infty} \frac{(r-2)(r-1)(2|p|+r)(2|p|+r-1)}{(2|p|+2 r-1)(2|p|+2 r-3)(2|p|+2 r+1)} \frac{(2|p|+r-1) !}{(r-1) !} d_{r}^{|p| n} d_{r-3}^{|p-1| n^{\prime}} \\
& +\sum_{r=0,1}^{\infty} \frac{(r+1)(r+2)(2|p|+r+2)(2|p|+r+3)}{(2|p|+2 r+1)(2|p|+2 r+3)(2|p|+2 r+5)} \frac{(2|p|+r+1) !}{(r+1) !} d_{r}^{|p| n} d_{r+1}^{p-1 \mid n^{\prime}} \\
& \left.+\sum_{r=2,1}^{\prime}\left(\frac{r+1}{2|p|+2 r+1}\right)^{2} \frac{r(2|p|+r+1)}{2|p|+2 r+3} \frac{(2|p|+r+1) !}{(r+1) !} d_{r}^{|p|, n} d_{r-1}^{p-1 \mid, n^{\prime}}\right], \quad\left|n-n^{\prime}\right|=\text { even, } \quad p \leq 0, \\
& =-2\left[\sum_{r=2,1}^{\infty}\left(\frac{2|p|+r}{2|p|+2 r+1}\right)^{2} \frac{1}{2|p|+2 r-1} \frac{(2|p|+r-1) !}{(r-1) !} d_{r}^{|p| n} d_{r+1}^{|p-1| n^{\prime}}\right. \\
& +\sum_{r=2,1}^{\prime} \frac{(2|p|+r)(r-1)}{(2|p|+2 r-3)(2|p|+2 r-1)(2|p|+2 r+1)} \frac{(2|p|+r-1) !}{(r-1) !} d_{r}^{p \mid n} d d_{r-1}^{|p-1| n^{\prime}} \\
& +\sum_{r=0,1}^{\prime} \frac{(2|p|+r+2)(r+1)}{(2|p|+2 r+1)(2|p|+2 r+3)(2|p|+2 r+5)} \frac{(2|p|+r+1) !}{(r+1) !} d_{r}^{p \mid n} d d_{r+3}^{|p-1| n^{\prime}} \\
& \left.+\sum_{r=0,1}^{\prime}\left(\frac{r+1}{2|p|+2 r+1}\right)^{2} \frac{1}{2|p|+2 r+3} \frac{(2|p|+r+1) !}{(r+1) !} d_{r}^{p \mid n} d_{r+1}^{|p-1| n^{\prime}}\right], \quad\left|n-n^{\prime}\right|=\text { even, } \quad p>0 \text {, } \\
& H_{n n^{\prime}, 1}^{p}=p \int_{0}^{\pi} S_{|p| n} S_{|p| n^{\prime}} \sin \theta d \theta=0, \quad\left|n-n^{\prime}\right|=\text { odd, } \quad 2 p \sum_{r=0,1}^{\infty} \frac{1}{2|p|+2 r+1} \frac{(2|p|+r) !}{r !} d_{r}^{|p| n} d_{r}^{|p| n^{\prime}}, \quad\left|n-n^{\prime}\right|=\text { even, }
\end{aligned}
$$

and

$$
\begin{aligned}
H_{n n^{\prime}, 2}^{p}= & \overline{\cos ^{2} \theta} H_{n n^{\prime}, 1}^{p}=p \int_{0}^{\pi} S_{|p| n} S_{|p| n^{\prime}} \sin \theta \cos ^{2} \theta d \theta=0, \quad\left|n-n^{\prime}\right|=\text { odd, } \\
= & 2 p\left[\sum_{r=2,1}^{\infty} \frac{1}{2|p|+2 r-1}\left(\frac{2|p|+r}{2|p|+2 r+1}\right)^{2} \frac{(2|p|+r-1) !}{(r-1) !} d_{r}^{p \mid n} d_{r}^{p \mid n^{\prime}}\right. \\
& +\sum_{r=2,3}^{\prime} \frac{(r-1)(2|p|+r)}{(2|p|+2 r-3)(2|p|+2 r-1)(2|p|+2 r+1)} \frac{(2|p|+r-1) !}{(r-1) !} d_{r}^{|p| n} d_{r-2}^{|p| n^{\prime}} \\
& +\sum_{r=0,1}^{\prime} \frac{(r+1)(2|p|+r+2)}{(2|p|+2 r+1)(2|p|+2 r+3)(2|p|+2 r+5)} \frac{(2|p|+r+1) !}{(r+1) !} d_{r}^{|p| n} d_{r+2}^{|p| n^{\prime}} \\
& \left.+\sum_{r=0,1}^{\infty} \frac{1}{2|p|+2 r+3}\left(\frac{r+1}{2|p|+2 r+1}\right)^{2} \frac{(2|p|+r+1) !}{(r+1) !} d_{r}^{|p| n} d_{r}^{|p| n^{\prime}}\right], \quad\left|n-n^{\prime}\right|=\text { even, }
\end{aligned}
$$


where $d_{r}^{|p| n}$, s are the expansion coefficients of angular functions $S_{m n}$ in spheroidal coordinates [33], and the superscript prime sign in the sum symbol indicates a series summation starting from $0(1)$ over the even (odd) indices of $r$ when $(n$ $-m$ ) is even (odd).

Equations (13) and (14) are also valid for a circularly or an elliptically polarized incident beam when the BSCs are reevaluated. For example, to calculate the torque exerted by a circularly polarized beam, the relevant coefficients $G_{n, T E}^{p, \text { circular }}$ and $G_{n, T M}^{p, \text { circular }}$ should be used. They can be obtained from the following superposition of BSCs evaluated for linearly polarized beams:

$$
\begin{aligned}
& G_{n, T E}^{p, \text { circular } \pm}=\frac{1}{\sqrt{2}}\left(G_{n, T E}^{p} \mp i G_{n, T M}^{p}\right), \\
& G_{n, T M}^{p, \text { circular } \pm}=\frac{1}{\sqrt{2}}\left(G_{n, T M}^{p} \pm i G_{n, T E}^{p}\right),
\end{aligned}
$$

where the superscript $+(-)$ of the BSCs denotes the two linear polarizations shifted in phase by $\pm \pi / 2$ so that right (left) circular polarization is produced.

Note that the torque components expressed by Eqs. (13) and (14) are normalized in such a way that the beam intensity at the focal point $I_{0}$, which can be related to the beam power $P$ and the beam shape confinement parameter $s$ by $I_{0}=2 P /\left[\pi \omega_{0}^{2}\left(1+s^{2}+1.5 s^{4}\right)\right]$ for a circular Gaussian beam [34], is omitted. It is needed for torque evaluation when a realistic beam is used.

\section{NUMERICAL CALCULATIONS}

Numerical procedures have been developed to implement the theory presented in the preceding section. As a check of the analytical solution, results have been compared to those obtained from direct numerical quadratures of Eqs. (8)-(10), and a complete agreement was found. After this verification, numerical calculations were made for both linearly and circular polarized Gaussian beams. We assumed the beam is produced by a cw Ti:sapphire laser $\left(\lambda_{0}=0.785 \mu \mathrm{m}\right)$ and focused to a waist radius of $\omega_{0}=1.0 \mu \mathrm{m}$. It is incident on a hard polystyrene particle of refractive index $M^{\prime}=1.573$ $+6.0 \times 10^{-4} i[35]$ surrounded by air $\left(M_{0}=1.0\right)$, or a single red blood cell (RBC) of refractive index $M^{\prime}=1.38+1.0$ $\times 10^{-5} i$ [36] embedded in a buffer solution with osmolarity adjusted to $\sim 130$ milliosmoles so that its refractive index is $M_{0}=1.335$ [8]. All the numerical results presented in this section are for the beam power $P=1 \mathrm{~W}$.

The most important step in GLMT calculations concerns the beam description and beam shape coefficients evaluation. For a loosely focused Gaussian beam, Davis' first-order corrected expressions [37] are a highly accurate approximation to a solution to Maxwell's equations, thereby sufficient for the beam description. In this case, the localized beam model $[38,39]$ can provide an accurate and efficient in BSC evaluation $[40,41]$. When the beam is focused to the same order as the wavelength $\left(\omega_{0} \sim \lambda\right.$, our case $)$, the first-order description becomes less accurate. In this case, Barton and Alexander's fifth-order beam description was used instead in our calcula-

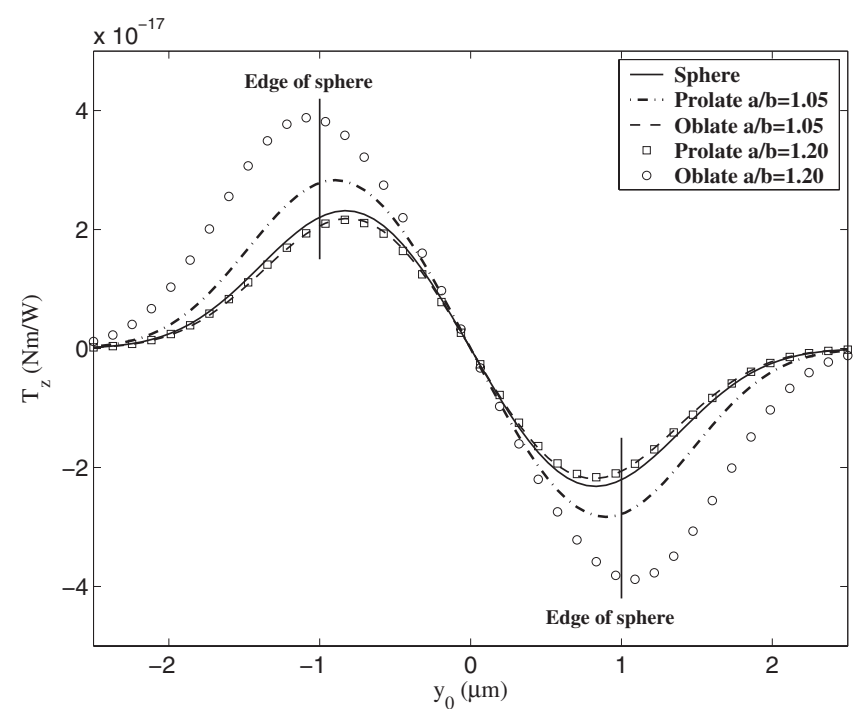

FIG. 2. Torque about the $z$ axis $\left(T_{\mathrm{z}}\right)$ versus the location of the beam center along $y$ axis $\left(y_{0}\right)$. A linearly polarized Gaussian beam of wavelength $\lambda_{0}=0.785 \mu \mathrm{m}$ and waist radius $\omega_{0}=1.0 \mu \mathrm{m}$ is incident on a polystyrene sphere of radius $r_{\mathrm{s}}=1.0 \mu \mathrm{m}$, two prolate spheroids of axis ratios $a / b=1.05$ and 1.20, and two oblate ones of the same ratios. These particles have the identical volume $V$ $=4.19 \mu \mathrm{m}^{3}$ and refractive index $M^{\prime}=1.573+6.0 \times 10^{-4} i$. The incidence and polarization angles of the beam are $90^{\circ}$ and $0^{\circ}$, respectively $\left(\left|\Theta_{\text {bd }}\right|=90^{\circ}\right.$ and $\left.\Phi_{\text {bd }}=0^{\circ}\right)$.

tion [34]. And we find the quadrature method [42] is more practical to evaluate the BSCs for such a tightly focused beam.

\section{A. Linearly polarized beam: Off-axis incidence}

First, we explore the influence the particle's location inside the beam has on the torque it receives. The particles considered here are two prolate polystyrene spheroids of axis ratios $a / b=1.05$ and 1.20 , two oblate ones of these ratios, and a sphere of radius $r_{\mathrm{s}}=1.0 \mu \mathrm{m}$. They are in air and all have the same volume $V=\frac{4}{3} \pi r_{s}^{3}=4.19 \mu \mathrm{m}^{3}$. The beam is assumed to be polarized in the $O_{\mathrm{P}^{-}} x z$ plane $\left(\Phi_{\mathrm{bd}}=0^{\circ}\right)$. It illuminates these spheroids vertically, i.e., side on $\left(\Theta_{\mathrm{bd}}=90^{\circ}\right)$. The beam center moves along the $y$ axis in the equatorial plane of the spheroid. In this case, the beam plays a role similar to a thin pencil of light that carries momentum. When it hits a spheroid, a radiation force is exerted on the particle along or opposite to the beam propagation direction. When the force is applied off center on the particle, there is a leverarm associated with the force and the orbital angular momentum can be successfully transferred to the particle through absorption, resulting in an orbit-absorption torque. Such a torque is about the $z$ axis in the current case. It is observed in Fig. 2 that the $T_{z}-y_{0}$ curves for a sphere and the spheroids have a similar shape. As the beam center moves along the $y$ axis, the lever-arm increases, and the torque correspondingly increases. Maxima of the torque appear at the vicinity of $\left|y_{0}\right|=0.8 \mu \mathrm{m}$, near the edge of the particle (see the labels in Fig. 2). Further increase in $y_{0}$ causes less beam light flux incident on the particle and less angular momentum is trans- 


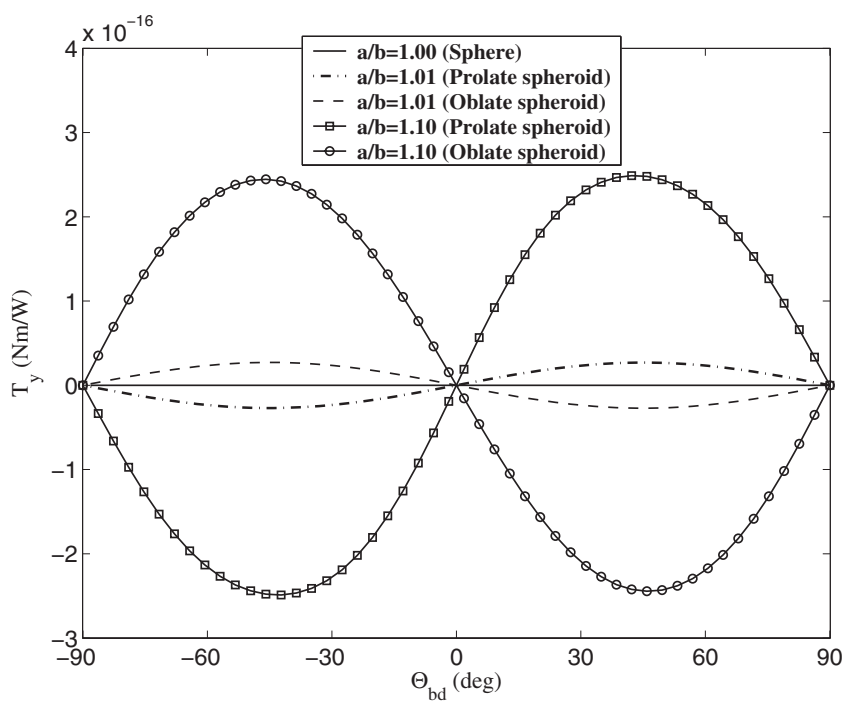

FIG. 3. Torque about the $y$ axis $\left(T_{\mathrm{y}}\right)$ versus the incident angle $\left(\Theta_{\text {bd }}\right)$ when a linearly polarized Gaussian beam of waist radius $\omega_{0}$ $=1.0 \mu \mathrm{m}$ and wavelength $\lambda_{0}=0.785 \mu \mathrm{m}$ is incident on a sphere and two spheroids of axis ratios $a / b=1.01$ and 1.10. The spheroids have the same volume as the sphere of radius $r_{\mathrm{s}}=1.0 \mu \mathrm{m}$. Their refractive index is $M^{\prime}=1.573+6.0 \times 10^{-4} i$. The beam center coincides with that of the particle and the beam polarization angle is $0^{\circ}$ $\left(\Phi_{\mathrm{bd}}=0^{\circ}\right)$. Note that in this figure $T_{\mathrm{y}}$ is zero for the sphere $\left(T_{\mathrm{y}}\right.$ $=0)$.

ferred, so the torque correspondingly decreases. In addition, a symmetry relation of the torque with respect to the point $\left(T_{z}=0, y_{0}=0\right)$ can be observed, as it should.

For a given $y_{0}$, we did not find a monotonic relationship between $T_{z}$ and $a / b$ during the deformation of the sphere to a prolate or oblate spheroid of axis ratio 1.20. This is because, even for a sphere, the absorption efficiency as a function of size parameter is a very rapidly varying function (see Fig. 7.2 on p. 170 of Ref. [43]). The situation for a spheroid would be similar when its size parameter defined by axis ratio and semimajor axis length, $C_{\mathrm{I}}=k_{\mathrm{I}} a\left[(a / b)^{2}-1\right]^{1 / 2}$ varies as the axis ratio increases. Therefore looking for trends in data as a function of $a / b$ or $C_{\mathrm{I}}$ will be complicated when the particle's refractive index greatly differs from that of the surrounding medium.

\section{B. On-axis beam incidence: Effect of incident angle}

Second, we investigate the influence of the incident angle on the torque. The beam center is set to coincide with that of the particle so that the beam is incident on axis. Torques are calculated for the incident angle increasing from $-90^{\circ}$ to $90^{\circ}$ $\left(-90^{\circ} \leq \Theta_{\mathrm{bd}} \leq 90^{\circ}\right)$. The particles considered here are two prolate spheroids and two oblate ones of axis ratios 1.01 and 1.10. They still have the same volume as a sphere of radius $r_{\mathrm{s}}=1.0 \mu \mathrm{m}$. Since there are no torque components about the $x$ and $z$ axes $\left(T_{\mathrm{x}}=T_{\mathrm{z}}=0\right)$ in this case, only $T_{\mathrm{y}}$ is plotted in Fig. 3. It can be observed that at a positive (negative) incident angle, there exists a positive (negative) torque causing counterclockwise (clockwise) rotation of the prolate spheroid about the $y$ axis. For an oblate spheroid, the direction of rotation is the opposite. Since during the rotation the incident angle gradually decreases for the prolate spheroid but increases for the oblate one, the particle "finds" its stable balance orientation corresponding to end-on incidence of the beam on the prolate spheroid $\left(\Theta_{\mathrm{bd}}=0^{\circ}\right)$ and side-on incidence on the oblate one $\left(\left|\Theta_{b d}\right|=90^{\circ}\right)$. Mathematically, the stable orientation $\Theta_{\mathrm{s}}$ is determined by

$$
T_{y}\left(\Theta_{\mathrm{s}}\right)=0,\left.\quad \frac{\partial T_{y}}{\partial \Theta_{\mathrm{bd}}}\right|_{\Theta_{\mathrm{bd}}=\Theta_{\mathrm{s}}}>0 .
$$

The torque found for these slightly absorbing spheroids is dominated by a reaction force which actually does not require the particles to be absorbing. To explain its physical mechanism, we develop in Appendix A the geometrical optics (GO) method in which the focused Gaussian beam illuminating a large spheroid is treated as a single ray. When it is refracted into the spheroid, the change of propagation direction produces a change of momentum of the reflected and transmitted rays and brings in the reaction force of order $p$ $=0$. When it is refracted out, the reaction force of order $p$ $=1$ is contributed. Reaction forces of higher orders are from the rays experiencing $p-1$ internal reflections. As proved in Appendix A, the reaction force arising from both reflection and refraction is along or opposite to the outwardly directed normal vector to the surface of the particle. For the special case of a sphere, this surface normal is along the direction of the lever-arm $\boldsymbol{R}$ so that the reaction force torques caused by both reflection $\left(\boldsymbol{R}_{p} \times \boldsymbol{F}_{\mathrm{p}}^{R}\right)$ and refraction $\left(\boldsymbol{R}_{p} \times \boldsymbol{F}_{p}^{T}\right)$ are equal to zero for all orders. For a spheroid, however, the surface normal does not coincide with the lever arm in direction unless an on-axis spheroid is illuminated by an end-on or side-on incident beam (here the term "on-axis" means the center of spheroid is on the beam axis).

In the current case of an on-axis spheroid illuminated by an oblique incident beam, torques of orders $p=0$ and 1 evaluated by $\boldsymbol{R}_{0} \times\left(\boldsymbol{F}_{0}^{R}+\boldsymbol{F}_{0}^{T}\right)$ and $\boldsymbol{R}_{1} \times\left(\boldsymbol{F}_{1}^{R}+\boldsymbol{F}_{1}^{T}\right)$, respectively, are found to have the leading contribution to the torque. They have the same sign when the spheroid is not extremely deformed from sphere, say $1.0 \leq a / b \leq 2.0$ for $\operatorname{Re}(M)$ $=1.573$. Since the normal to an oblate spheroid lies on the opposite side of the incidence direction as does the normal for a prolate spheroid, an opposite direction of rotation is induced, as indicated by Fig. 3 .

In the addition to the dominating contribution from the reaction force induced torque, the orbit-absorption torque also appears on an absorbing spheroid even when it is onaxis located. To explain the appearance of the orbitabsorption torque, we trace two rays contained in the Gaussian beam and symmetric about the beam axis. Since for an on-axis spheroid, the lever-arms $(\boldsymbol{R})$ associated with photon absorption induced forces $(d \boldsymbol{p} / d t)$ along the optical paths $L$ of these two rays are not equal, a residual torque evaluated by $\Delta \boldsymbol{T}=\int_{L_{1}} d \boldsymbol{R}_{1} \times \frac{d p_{1}}{d t}+\int_{L_{2}} d \boldsymbol{R}_{2} \times \frac{d \boldsymbol{p}_{2}}{d t} \neq \mathbf{0}$ is induced. For a sphere, however, the numbers of absorbed photons from these two rays are exactly identical and the lever arms are symmetric about the beam axis along the internal light path so that the two induced torques counteract each other $(\Delta \boldsymbol{T}$ $=\mathbf{0})$. To demonstrate the existence and effect of this orbit- 


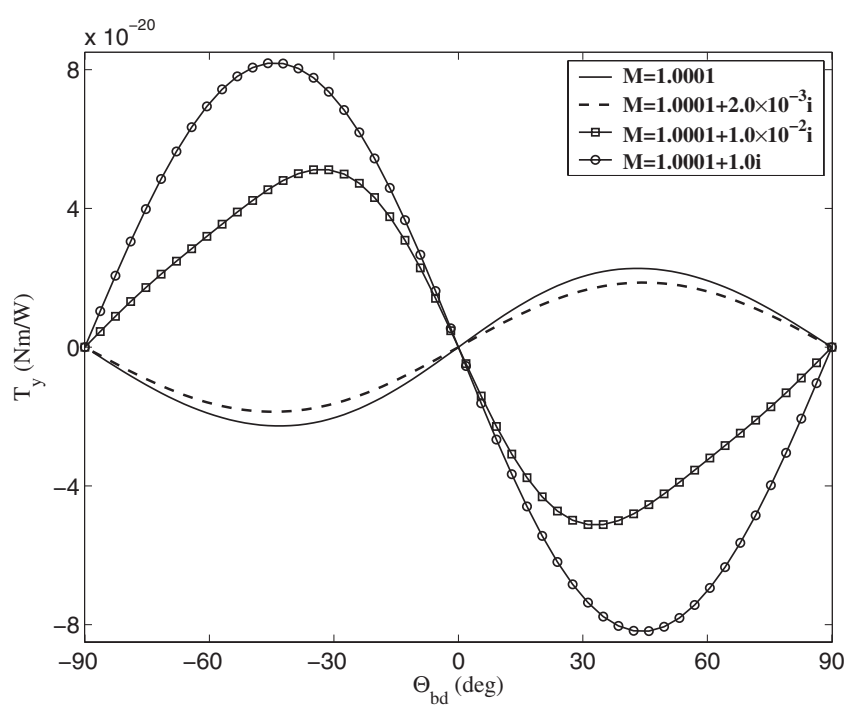

FIG. 4. Torque about the $y$ axis $\left(T_{\mathrm{y}}\right)$ versus the incident angle $\left(\Theta_{\text {bd }}\right)$ when a linearly polarized Gaussian beam of waist radius $\omega_{0}$ $=1.0 \mu \mathrm{m}$ and wavelength $\lambda_{0}=0.785 \mu \mathrm{m}$ is incident on a prolate spheroid of axis ratio $a / b=1.10$ and volume equal to that of a sphere of radius $r_{\mathrm{s}}=1.0 \mu \mathrm{m}$. The real part of its refractive index is set to 1.0001 and the imaginary part increases from $0,2.0 \times 10^{-3}$, $1.0 \times 10^{-2}$, and then to 1.0. The beam has its center coinciding with that of the particle and its electric field $\mathbf{E}$ is polarized in the $O_{\mathrm{P}^{-}} x y$ plane. Note that for clarity the results for the spheroid of $M$ $=1.0001+1.0 i$ has been divided by 600 .

absorption torque for an on-axis spheroid, we take a prolate spheroid of axis ratio 1.10 and with volume equal to that of a sphere of radius $r_{\mathrm{s}}=1.0 \mu \mathrm{m}$ as an example. The real part of its refractive index is set to 1.0001 so that the angular momentum change due to refraction and reflection is small and the contribution of the reaction force torque can be effectively reduced. Meanwhile, we let the imaginary part of the particle's refractive index increase from 0 to $2.0 \times 10^{-3}, 1.0$ $\times 10^{-2}$, and then to 1.0. It can be observed from Fig. 4 that with the increase of particle's absorption capacity, the torque first decreases and then flips its sign. Similar effects also occur for an oblate spheroid. This means there exists an orbit-absorption torque that rotates the spheroid in a direction opposite to that caused by reaction force induced torque. To completely overcome the torque resulting from the reaction force, the absorption of the spheroid should reach a certain level so that the rotation is dominated by the orbitabsorption torque. The transition point $\operatorname{Im}\left(M_{\mathrm{t}}\right)$ is found to be greatly dependent on the real part of the particle's refractive index. For the current case of $\operatorname{Re}(M)=1.0001$, its value is found to be $5.45 \times 10^{-3}$ and $5.83 \times 10^{-3}$ for prolate and oblate spheroids, respectively. When $\operatorname{Re}(M)$ increases to 1.573 , its value increases to $9.16 \times 10^{-2}$ and $9.04 \times 10^{-2}$ for prolate and oblate spheroids, respectively. Note that when the imaginary part of the particle's refractive index is increased to be sufficiently large so that the particle tends to be totally reflecting, the reaction force dominates again the torque. And the rotation still becomes reverse since the torque caused by the reflected rays at the order $p=0$ and evaluated by $\boldsymbol{R}_{0}$ $\times \boldsymbol{F}_{0}^{R}$ has a direction opposite to that of the torque caused by the refracted rays $\left(\boldsymbol{F}_{0}^{T} \rightarrow \mathbf{0}\right.$ in this case). But in our former

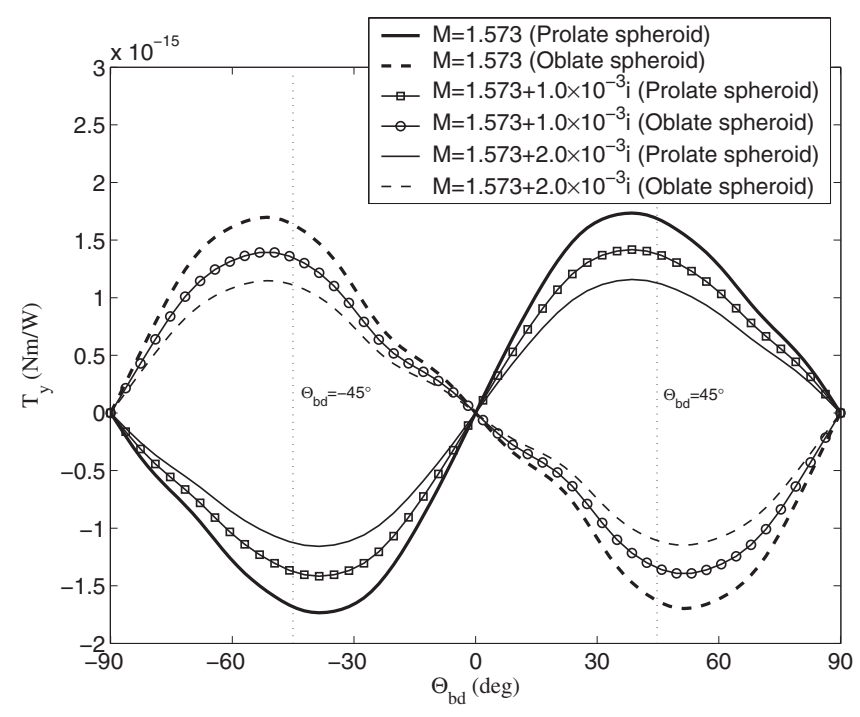

FIG. 5. Torque about the $y$ axis $\left(T_{\mathrm{y}}\right)$ versus the incident angle $\left(\Theta_{\mathrm{bd}}\right)$ when a linearly polarized Gaussian beam of waist radius $\omega_{0}$ $=1.0 \mu \mathrm{m}$ and wavelength $\lambda_{0}=0.785 \mu \mathrm{m}$ is incident on polystyrene prolate and oblate spheroids of axis ratio $a / b=1.10$ and volume equal to that of a sphere of radius $r_{\mathrm{s}}=5.0 \mu \mathrm{m}$. The beam has its center coinciding with that of the particle and its electric field $\mathbf{E}$ is polarized in the $O_{\mathrm{P}^{-}} x y$ plane. The particles are suspended in air and have real refractive index 1.573 while the imaginary part increases from 0 to $1.0 \times 10^{-3}$, and then to $2.0 \times 10^{-3}$.

case of the slightly absorbing spheroid (see Fig. 3), $\left|\boldsymbol{F}_{0}^{T}\right|$ is larger than $\left|\boldsymbol{F}_{0}^{R}\right|$ and hence dominates the reaction force torque of order $p=0$.

As a qualitative verification of GLMT for micrometer scale particles, the GO model developed in Appendix A is used. Ideally, the GO model requires the size of spheroid to be much larger than the beam waist so that the curvature of the spheroidal surface in $y$ direction can be neglected and the three dimensional problem can be simplified to two dimensions. However, an extremely large size is beyond the capability of our current computational program developed for GLMT. Therefore to compare the results obtained by GO and GLMT, the torque is calculated for spheroids with volume equal to that of a sphere of not very large radius $r_{\mathrm{s}}$ $=5.0 \mu \mathrm{m}$. They are assumed to have an axis ratio $a / b$ $=1.10$ and refractive indices $M^{\prime}=1.573,1.573+1.0 \times 10^{-3} i$, and $1.573+2.0 \times 10^{-3} i$. A Gaussian beam of waist radius $\omega_{0}=1.0 \mu \mathrm{m}$ and electric field $\mathbf{E}$ lying in the $O_{\mathrm{P}^{-}} x y$ plane is used in the GLMT calculation. In the GO calculation such a beam is approximated by a single ray and the maximum order of ray tracing is taken to be $p_{\max }=20$. The results are plotted in Fig. 5 for GLMT and Fig. 6 for GO. Both calculations predict a positive torque for a prolate (oblate) spheroid at positive (negative) incident angles. The maximum torque appears at an incident angle smaller (larger) than $45^{\circ}$ for a prolate (oblate) spheroid. Such an angle is found corresponding to the maximal momentum change of transmitted rays of orders $p=0$ and $p=1$, which contribute most to the final torque. More calculations show that further increasing the axis ratio will shift the maximal torque on a prolate and/or oblate spheroid toward even smaller (larger) incident angles. Figures 5 and 6 also exhibit the decrease of the 


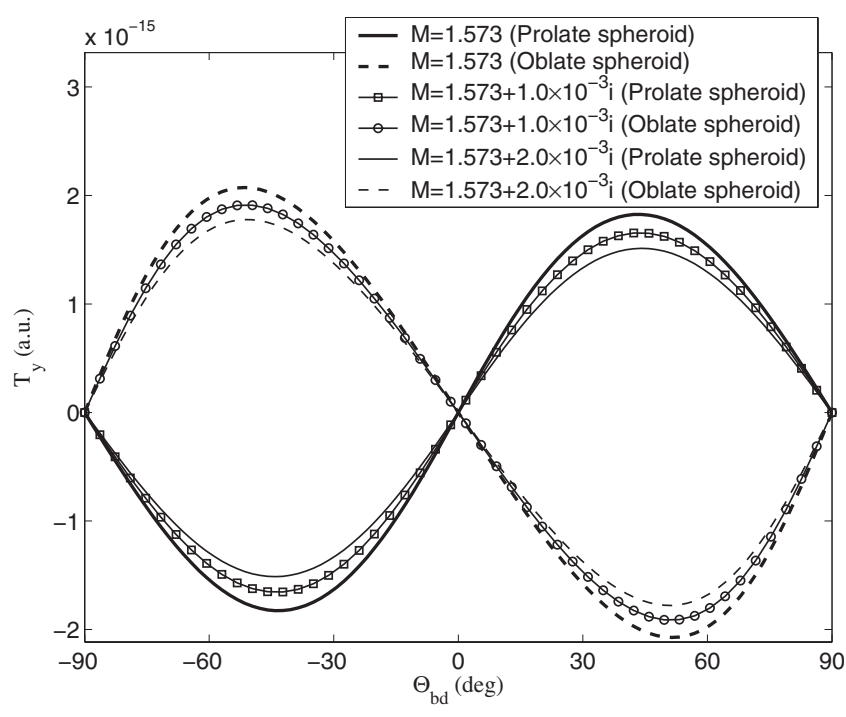

FIG. 6. Torque about the $y$ axis $\left(T_{\mathrm{y}}\right)$ versus the incident angle $\left(\Theta_{\text {bd }}\right)$ when a light ray of wavelength $\lambda_{0}=0.785 \mu \mathrm{m}$ is incident on the polystyrene prolate and oblate spheroids of axis ratio $a / b$ $=1.10$ and volume equal to that of a sphere of radius $r_{\mathrm{s}}=5.0 \mu \mathrm{m}$. The light ray is directed toward the particle center $O_{\mathrm{P}}$ and it has its electric field $\mathbf{E}$ polarized in the $O_{\mathrm{P}^{-}} x y$ plane. The particles are suspended in air and have real refractive index 1.573 while the imaginary part increases from 0 to $1.0 \times 10^{-3}$, and then to $2.0 \times 10^{-3}$. Torque in the figure has arbitrary units (a.u.).

torque due to increasing absorption. There are two reasons for this phenomenon. First, increasing the imaginary part of a slightly absorbing particle's refractive index means more intensity attenuated in the spheroid so that the rays of order $p=1$ contribute less and the reaction force induced torque decreases accordingly. Second, the orbital momentum transferred from the absorbed photons induces an orbit-absorption torque of opposite sign to that caused by the reaction force. Therefore the overall torque decreases further. The orbitabsorption torque is not included in our GO model, therefore the decrease of torque predicted by GO in Fig. 6 is less remarkable than that predicted by GLMT in Fig. 5 .

The stable orientations found in the preceding examples for slightly absorbing spheroids only pertain to the particles of size approaching or larger than the wavelength. It does not pertain to the nanometer scale particles of size much smaller than the wavelength. An extremely small spheroid embedded in an external electric field $\mathbf{E}$ polarizes with the induced dipole moment $\mathbf{P}$, but usually not along the direction of the external field unless the $\mathbf{E}$ field direction is along one of the particle's symmetry axes. A polarization induced torque ( $\mathbf{T}$ $=\mathbf{P} \times \mathbf{E})$ appears in this case and tends to orient the major axis of the spheroid in the direction of $\mathbf{E}$ to minimize the stored energy in the field [44]. As an example, we give in Fig. 7 the $T_{y}-\Theta_{\text {bd }}$ curves calculated by GLMT for two prolate and two oblate spheroids with volume equal to that of a sphere of radius $r_{\mathrm{s}}=10 \mathrm{~nm}$. According to the criterion expressed by Eq. (21), the stable orientations are found to be characterized by the incident angle $\left|\Theta_{\mathrm{bd}}\right|=90^{\circ}$ for a prolate spheroid and by $\Theta_{\text {bd }}=0^{\circ}$ for an oblate one. As indicated by the dotted lines in Fig. 7, these calculations are confirmed by dipole method whose theoretical development is described in

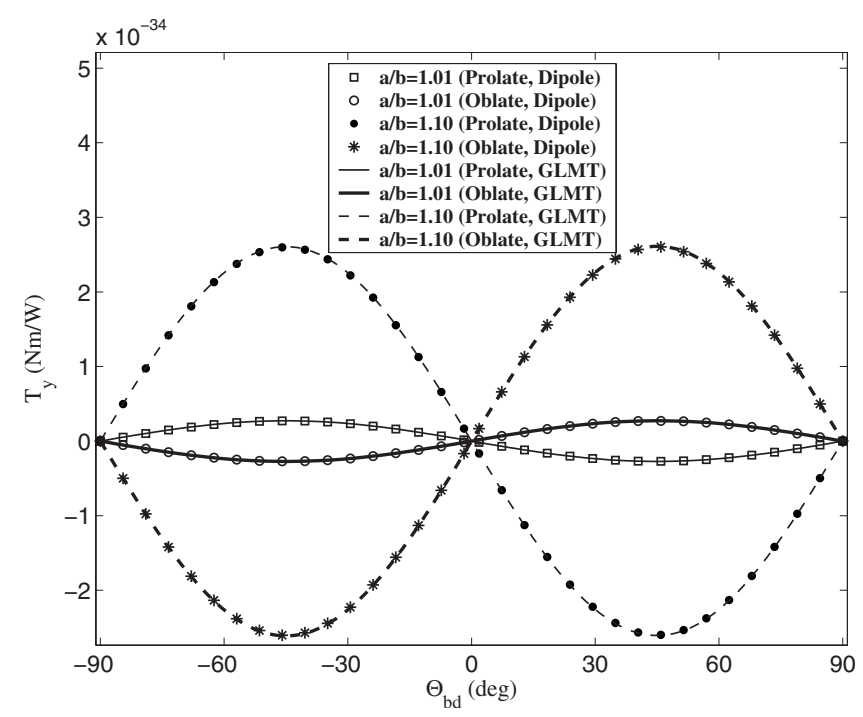

FIG. 7. Torque about the $y$ axis $\left(T_{\mathrm{y}}\right)$ versus the incident angle $\left(\Theta_{\mathrm{bd}}\right)$ when a linearly polarized plane wave of wavelength $\lambda_{0}$ $=0.785 \mu \mathrm{m}$ is incident on the polystyrene spheroids of refractive index $M^{\prime}=1.573+6.0 \times 10^{-4} i$ and axis ratios $a / b=1.01$ and 1.10 . These spheroids have volume equal to that of a sphere of radius $r_{\mathrm{s}}=10 \mathrm{~nm}$. The plane wave has the polarization angle $0^{\circ}\left(\Phi_{\mathrm{bd}}\right.$ $\left.=0^{\circ}\right)$. The incident electric field magnitude $\left|\mathrm{E}_{0}\right|$ is assumed to be unity $\left(\left|\mathrm{E}_{0}\right|=1\right)$.

detail in Appendix B. Numerical calculations show that the dipole method has errors less than $1.0 \%$ for the particles of size about 20 times smaller than the incident wavelength.

Since the comparison of Fig. 3 to Fig. 7 has indicated that spheroids of nanometer scale and micrometer scale sizes have different stable orientations, it is interesting to know the transition size $r_{\mathrm{s}, \mathrm{t}}$. In our current case using a linearly polarized Gaussian beam of waist radius $\omega_{0}=1.0 \mu \mathrm{m}$, an average value of $r_{\mathrm{s}, \mathrm{t}}$ for the prolate polystyrene spheroids of axis ratio $1.0 \leq a / b \leq 2.0$ and refractive index $M^{\prime}=1.573+6.0 \times 10^{-4} i$ is found to be about $0.2 \mu \mathrm{m}$.

\section{On-axis beam incidence: Effect of polarization angle}

To further explore the effect of the polarization induced torque, we examine the influence of the polarization angle $\Phi_{\text {bd }}$ on the torque at the stable orientation of the spheroid. First we discuss the case of oblate spheroids of micrometer scale size. When $\Phi_{\text {bd }}$ is not equal to $0^{\circ}$ or $\pm 90^{\circ}$, the induced dipole $\mathbf{P}$ of the spheroid is not along the direction of $\mathbf{E}$ field. Using the oblate spheroids of the same parameters as those in Fig. 3, we give in Fig. 8 the torque about the $x$ axis $\left(T_{x}\right)$ for the oblate spheroids of stable orientation characterized by $\Theta_{\text {bd }}=90^{\circ}$ (no torque components about $y$ and $z$ axes exist in this situation, namely $T_{\mathrm{y}}=T_{\mathrm{z}}=0$ ). It can be found that $T_{\mathrm{x}}$ rotates the particles to the stable orientation of the beam polarization angle $\left|\Phi_{\text {bd }}\right|=90^{\circ}$. Mathematically, this stable polarization angle $\Phi_{\mathrm{s}}$ is described by

$$
T_{x}\left(\Phi_{\mathrm{s}}\right)=0,\left.\quad \frac{\partial T_{x}}{\partial \Phi_{\mathrm{s}}}\right|_{\Phi_{\mathrm{bd}}=\Phi_{\mathrm{s}}}>0 .
$$

Our prediction is consistent with the experimental observation of Bayoudh et al. [45]. 


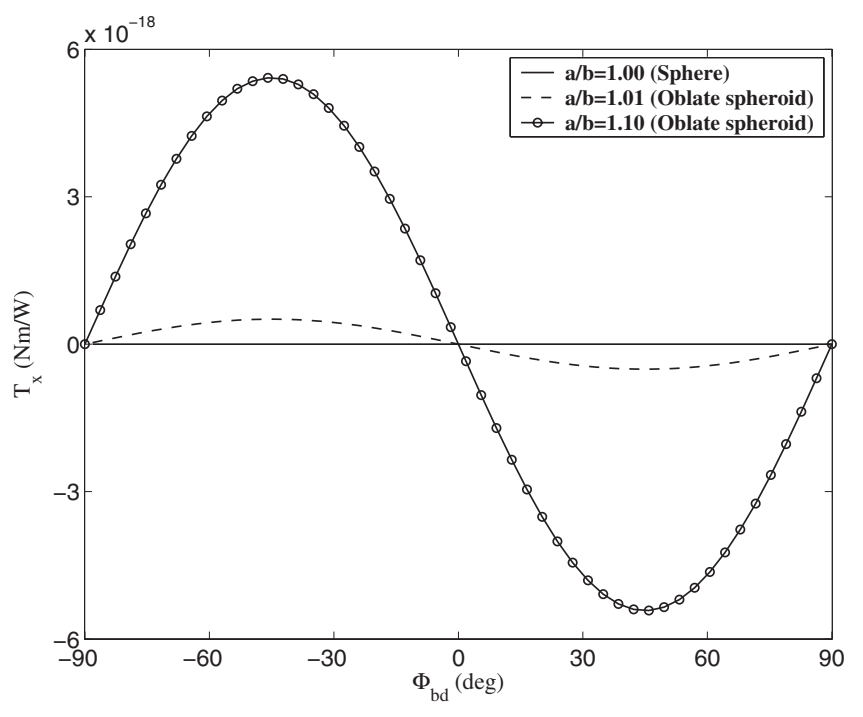

FIG. 8. Torque about the $x$ axis $\left(T_{\mathrm{x}}\right)$ versus the polarization angle $\left(\Phi_{\mathrm{bd}}\right)$ when a linearly polarized Gaussian beam is incident on a sphere and two oblate spheroids. The parameters of the beam and the particles are the same as in Fig. 3. But the incident angle is now fixed to be $90^{\circ}\left(\left|\Theta_{\mathrm{bd}}\right|=90^{\circ}\right)$. Note that $T_{\mathrm{x}}$ is zero for the sphere at all polarization angles $\left(T_{\mathrm{x}}=0\right)$.

Next we show in Fig. 9 the $T_{\mathrm{x}}-\Phi_{\mathrm{bd}}$ curves for two nanometer scale prolate spheroids of axis ratios $a / b=1.01$ and 1.10 , respectively, and having a volume equal to that of a sphere of radius $r_{\mathrm{s}}=10 \mathrm{~nm}$. They are vertically illuminated by the plane wave $\left(\Theta_{\mathrm{bd}}=90^{\circ}\right)$. According to Eq. (22), the stable polarization angle is found to be characterized by $\Phi_{\mathrm{bd}}=0^{\circ}$ for a prolate spheroid. These calculations are confirmed by a dipole method which gives the results plotted as dotted lines in Fig. 9.

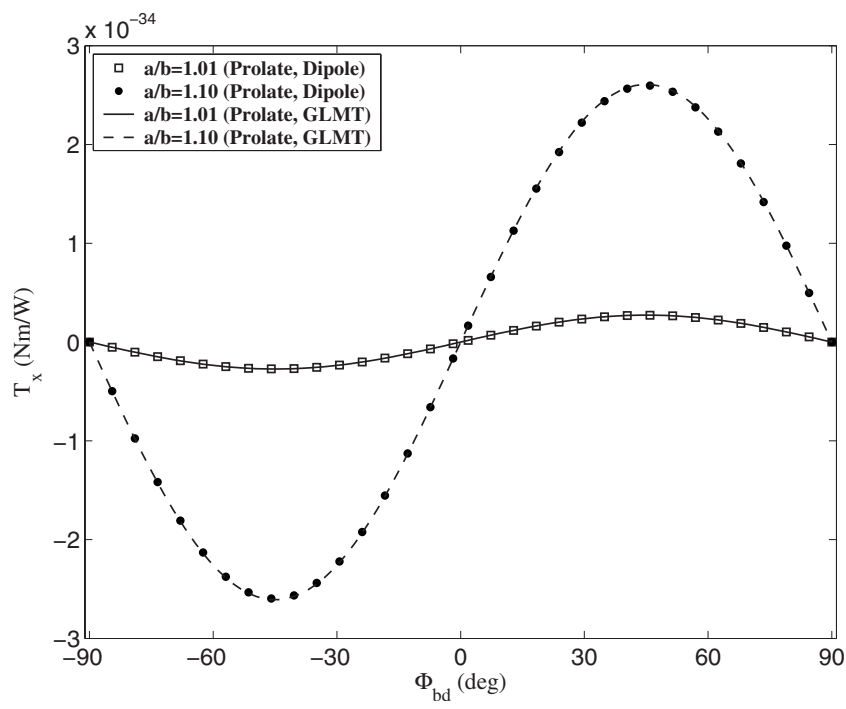

FIG. 9. Torque about the $x$ axis $\left(T_{\mathrm{x}}\right)$ versus the polarization angle $\left(\Phi_{\mathrm{bd}}\right)$ when a linearly polarized plane wave of wavelength $\lambda_{0}=0.785 \mu \mathrm{m}$ is incident on two prolate polystyrene spheroids of refractive index $M^{\prime}=1.573+6.0 \times 10^{-4} i$ and axis ratios $a / b=1.01$ and 1.10. The spheroids have volume equal to that of a sphere of radius $r_{\mathrm{s}}=10 \mathrm{~nm}$. The incident angle of the beam is fixed to be $90^{\circ}$ $\left(\left|\Theta_{\text {bd }}\right|=90^{\circ}\right)$.
Note that due to the rotational symmetry about the beam axis, polarization torque does not exist on a prolate spheroid of micrometer scale at the beam incident angle $\Theta_{b d}=0^{\circ}$ or on an oblate spheroid of nanometer scale at $\Theta_{\mathrm{bd}}=90^{\circ}$.

Thus we can end this part of the discussion by concluding that the torque exerted on an extremely small spheroid $\left(r_{\mathrm{s}} \ll \lambda\right)$ is dominated by the polarization induced torque which leads to the stable orientation $\left(\left|\Theta_{\mathrm{bd}}\right|=90^{\circ}, \Phi_{\mathrm{bd}}=0^{\circ}\right)$ and $\left(\Theta_{\mathrm{bd}}=0^{\circ}, \forall \Phi_{\mathrm{bd}}\right)$ for a prolate spheroid and for an oblate one, respectively. When the size of the particle increases to the geometrical optics limit and its absorption is slight, the reaction force induced torque becomes significant and leads to end-on (side-on) incidence of the beam on the prolate (oblate) spheroid. Finally, the stable orientation is characterized by $\left(\Theta_{\mathrm{bd}}=0^{\circ}, \forall \Phi_{\mathrm{bd}}\right)$ and $\left(\left|\Theta_{\mathrm{bd}}\right|=90^{\circ},\left|\Phi_{\mathrm{bd}}\right|=90^{\circ}\right)$ for a prolate spheroid and for an oblate one, respectively. Since completely opposite rotation directions are involved in the two limits, there exists a trade-off size $r_{\mathrm{s}, \mathrm{t}}$ in the transition region. In addition, increasing particle's absorption capacity to a certain level might cause the stable orientation of the spheroids of micrometer scale to be same as that found for the spheroids of nanometer scale.

\section{Circularly polarized beam incidence: Effect of axis ratio}

As discussed in the preceding subsection, the torque exerted by a linearly polarized beam aligns a spheroid to a given orientation. There should be some oscillations of the spheroid back and forth about the stable orientation. After a certain period, the oscillations are damped out by friction and the particle finally remains stationary inside the beam. However, when a circularly polarized beam is used the induced torque makes the spheroid rotate at the formerly stable orientations.

As an example, we simulated the "optical stretcher" employing two coaxial but two counterpropagating Gaussian beams to exert forces on a RBC. The beams are assumed right- and left-circularly polarized, respectively (though they are linearly polarized in actual experiments). The originally spherical RBC of radius $r_{\mathrm{s}}=3.0 \mu \mathrm{m}$ is embedded in a buffer solution of refractive index $M_{0}=1.335$. It is trapped where the beam cross sections are same and the local waist radius is $10 \%$ larger than that of the particle $\left(\omega=1.10 r_{s}\right)$ [8]. During the deformation its shape is approximated by a prolate spheroid of axis ratio $a / b$ growing from 1.0 to 1.5 . The changes of torque about the $z$ axis $\left(T_{z}\right)$ and absorption cross section $\left(C_{a b s}\right)$ are illustrated in Figs. 10 and 11, respectively. With the refractive index of the RBC approaching that of the medium $\left(M=1.03+1.0 \times 10^{-5} i\right)$, a monotonic increase of the torque during the deformation can be observed. Since the magnitude of torque depends on the number of absorbed photons carrying angular momentum, the torque curve exhibits the same shape as that of absorption cross section. Assuming the central beam intensity $I_{0}$ to be unity, the torque exerted by a circularly polarized beam can be related to the absorption cross section for the case of on-axis beam incidence $\left(\Theta_{\mathrm{bd}}=0\right.$ and $G_{n}^{p \text {,circular }}=0$ when $\left.p \neq \pm 1\right)$ by 


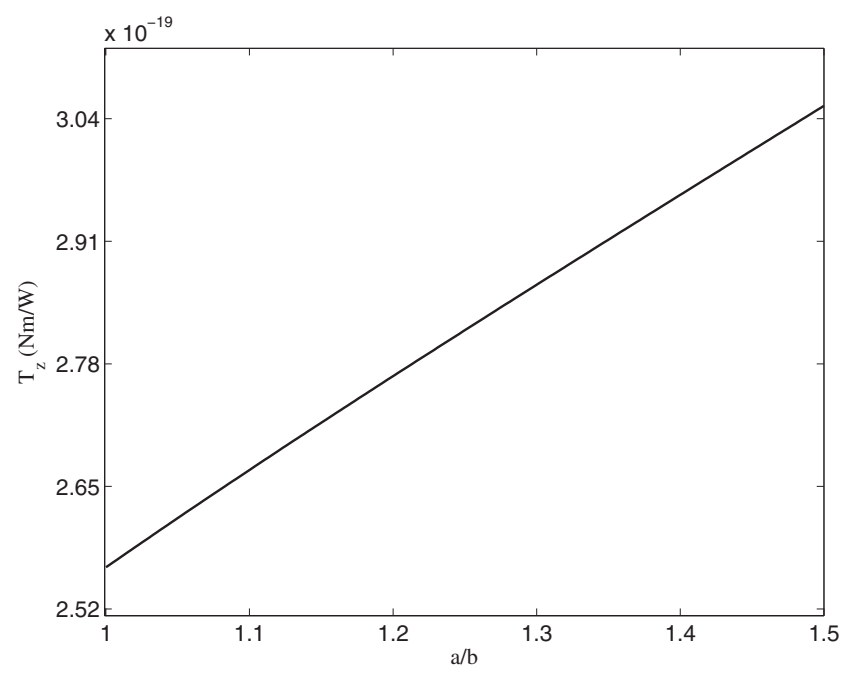

FIG. 10. Torque about the $z$ axis $\left(T_{\mathrm{z}}\right)$ versus axis ratio $(a / b)$ for a prolate spheroid. Two coaxial but counterpropagating Gaussian beams, which are right- and left-circularly polarized, respectively, stretch an originally spherical $\mathrm{RBC}$ of refractive index $M^{\prime}=1.38$ $+1.0 \times 10^{-5} i$ and radius $r_{\mathrm{s}}=3.0 \mu \mathrm{m}$. It is embedded in a buffer solution of refractive index $M_{0}=1.335$. The beams have the waist radius $\omega_{0}=1.0 \mu \mathrm{m}$. The $\mathrm{RBC}$ is located where the beam cross sections are same and the radius of local beam waist is $10 \%$ larger than that of the particle $\left(\omega=1.10 r_{s}\right)$. During the deformation, its axis ratio is assumed to increase from 1.0 to 1.5 . Note that in this figure, $T_{\mathrm{z}}$ is the contribution of each beam.

$$
T_{z}= \pm \frac{4 M_{0} \pi^{2}}{c k_{\mathrm{I}}^{3} \lambda_{\mathrm{I}}^{2}} C_{a b s}
$$

where + and - correspond to the right- and left-circularly polarized beams, respectively, and the absorption cross section $C_{a b s}$ can be obtained by replacing the BSCs in the following formula for a linearly polarized beam by those for a circularly polarized beam [Eqs. (19) and (20)]:

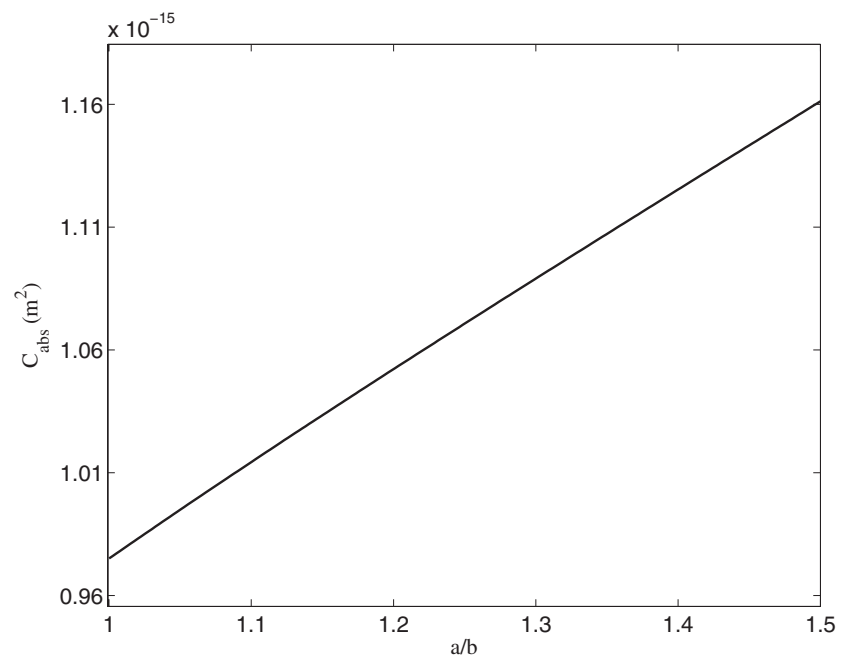

FIG. 11. Absorption cross section of the spheroid during deformation. Parameters of the beam and spheroid are the same as in Fig. 10.

$$
\begin{aligned}
C_{a b s}= & -\frac{\lambda^{2}}{\pi} \operatorname{Re}\left[\sum _ { p = - \infty } ^ { + \infty } \sum _ { n = | p | \neq 0 } ^ { + \infty } \sum _ { n ^ { \prime } = | p | \neq 0 } ^ { + \infty } \Pi _ { n n ^ { \prime } } ^ { p } \left(A_{n}^{p *} G_{n^{\prime}, T M}^{p}\right.\right. \\
& \left.\left.+B_{n}^{p *} G_{n^{\prime}, T E}^{p}+A_{n}^{p *} A_{n^{\prime}}^{p}+B_{n}^{p *} B_{n^{\prime}}^{p}\right)\right],
\end{aligned}
$$

where

$$
\begin{aligned}
\Pi_{n n^{\prime}}^{p}= & 0, \quad\left|n-n^{\prime}\right|=\text { odd, } \\
& \sum_{r=0,1}^{\infty} \frac{(|p|+r)(|p|+r+1)}{2|p|+2 r+1} \frac{(2|p|+r) !}{r !} d_{r}^{p \mid n} d_{r}^{p \mid n^{\prime}}, \\
& \left|n-n^{\prime}\right|=\text { even. }
\end{aligned}
$$

\section{E. Circularly polarized beam incidence: Effect of incident angle}

Next, we investigate the influence of the incident angle on the torque. The micrometer scale particles used here are the same as those used for Fig. 3 except that the beam is now right-circularly polarized and the particle has a purely real refractive index 1.573. In this case, the reaction force induced torque still dominates the rotation about the $y$ axis $\left[T_{y}\right.$, Fig. 12(b)] causing stability at end-on (side-on) incidence of the beam on the prolate (oblate) spheroid. But contrary to the case when a linearly polarized beam is used, an additional torque about the $x$ axis can also be observed in Fig. 12(a). This should be the polarization induced torque due to the temporal variation of the polarization direction of the electric field $\mathbf{E}$. Though the variation is quite rapid in time scale, a residual effect still occurs. This torque keeps a transparent oblate spheroid rotating at its stable orientation characterized by the beam incident angle $\left|\Theta_{\text {bd }}\right|=90^{\circ}$ (but not for a prolate spheroid at its stable orientation with $\Theta_{\mathrm{bd}}=0^{\circ}$ ). Its appearance has also been predicted by coupled dipole method developed for micropropeller driven by a circularly polarized beam [48]. It is noteworthy that the torque about the $z$ axis $\left(T_{z}\right)$ is zero for all incident angles. The reason is that when we decompose the local electric field $\mathbf{E}$ into components in the $x$ and $z$ directions, the spinning part about the $z$ axis actually induces no torque because of the spheroid's symmetry about this axis and its nonabsorption of light.

To generate a torque about the $z$ axis, the particle has to be absorbing so that the angular momentum of some incident photons is transferred to the spheroid via spin-absorption mechanism. It can be seen in Fig. 13(c) that $T_{z}$ appears when the imaginary part of the refractive index of the spheroids in the preceding example is increased to $6.0 \times 10^{-4}$. Moreover, at the stable orientation of an oblate spheroid $\left(\left|\Theta_{b d}\right|=90^{\circ}\right)$, comparison of the torque about the $x$ axis for an absorbing particle [Fig. 13(a)] to that for a transparent one [Fig. 12(a)] indicates that torques induced by polarization and spinabsorption rotate the particle toward a same direction in spite of their difference in magnitude.

As to absorbing spheroids of nanometer scale, all torque components occur as well when the particle is illuminated by an oblique incident beam. In Fig. 14 is illustrated an example 

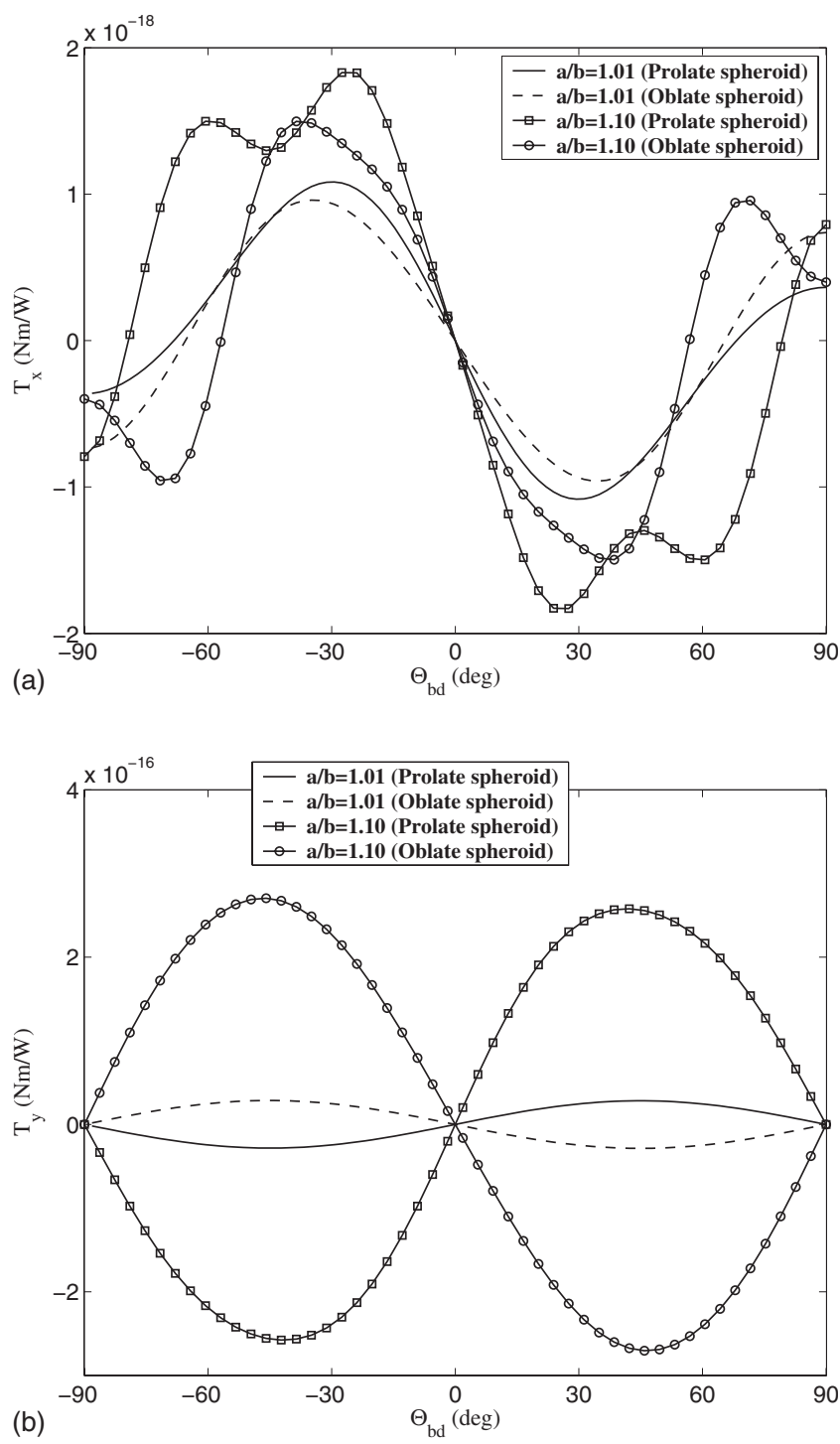

FIG. 12. Torque components $T_{\mathrm{x}}$ (a) and (b) $T_{\mathrm{y}}$ versus the incident angle $\left(\Theta_{\mathrm{bd}}\right)$ of the beam. The particles used here are same as those in Fig. 3, but their refractive indices are now purely real, $M^{\prime}=1.573$. And the beam is now right-circularly polarized.

for polystyrene spheroids with volume equal to that of a sphere of radius $r_{\mathrm{s}}=10 \mathrm{~nm}$. But in this case, the polarization induced torque tends to align a prolate (oblate) spheroid into the orientation characterized by $\left|\Theta_{\mathrm{bd}}\right|=90^{\circ}\left(\Theta_{\mathrm{bd}}=0^{\circ}\right)$. For a transparent prolate spheroid, a polarization induced torque will rotate the particle about the beam axis at its stable orientation.

For spheres of whatever size, however, there is no rotation about the $y$ axis $\left(T_{y}=0\right)$. They have to be absorbing to induce the torque components $T_{\mathrm{x}}$ and $T_{\mathrm{z}}$ which are related by the following equation:

$$
T_{x, \Theta_{\mathrm{bd}}}^{2}+T_{z, \Theta_{\mathrm{bd}}}^{2}=T_{z, \Theta_{\mathrm{bd}}=0^{\circ}}^{2}=T_{x, \Theta_{\mathrm{bd}}=90^{\circ}}^{2}
$$

Equation (26) indicates an overall rotation of the sphere about the beam axis at a constant angular velocity.

\section{CONCLUSION}

Within the framework of generalized Lorenz-Mie theory, the analytical solution to the radiation torque exerted on a spheroid by an arbitrarily shaped beam is derived. Numerical results indicate two types of torques not found for a sphere: the polarization induced torque and the reaction force induced torque. Their physical mechanisms are analyzed using geometrical optics for large spheroids and the dipole polarization method for small ones.

Due to these torques, a polarized Gaussian beam obliquely incident on a spheroid causes its rotation into a stable orientation. This orientation depends on the particle size relative to the incident wavelength as well as its refractive index. For both transparent and slightly absorbing spheroids, when its size approaches or is larger than the wavelength (e.g., micrometer scale spheroids) the stable orientation dominated by the reaction force indcued torque is associated with end-on incidence of the beam on a prolate spheroid and side-on incidence on an oblate spheroid. While for a particle of size much smaller than the wavelength (e.g., nanometer scale spheroids), the stable orientation is decided by the polarization indueced torque and corresponds to side-on incidence of the beam on a prolate spheroid and end-on incidence on an oblate one. Moreover, the polarization induced torque aligns the equatorial plane of a micrometer scale (nanometer scale) oblate (prolate) spheroid parallel (perpendicular) to the polarization direction of the $\mathbf{E}$ field at stable orientations. It should be noted that the stable orientation predicted for spheroids of micrometer scale might change to those found for spheroids of nanometer scale when particles increases its absorption to a certain level and the reaction force induced torque is dominated by an orbit-absorption torque rotating the on-axis spheroid in the opposite direction. The same effect can be found for a highly absorbing spheroid which totally reflects the incident light, bringing in a reaction force purely contributed by external reflection.

When illuminated by a circularly polarized beam, the stable orientation is still related to the beam incident angle $\Theta_{\mathrm{bd}}=0^{\circ}\left(\left|\Theta_{\mathrm{bd}}\right|=90^{\circ}\right)$ for a prolate (oblate) spheroid of micrometer scale and by $\left|\Theta_{\mathrm{bd}}\right|=90^{\circ}\left(\Theta_{\mathrm{bd}}=0^{\circ}\right)$ for a prolate (oblate) spheroid of nanometer scale. And the spinabsorption induced torque dominates the rotation of an absorbing spheroid about the beam axis at the final stable orientation. However, the rotation is dominated by polarization torque for two types of transparent spheroids: prolate ones of nanometer scale and oblate ones of micrometer scale.

\section{ACKNOWLEDGMENT}

This research is supported by Alexander von Humboldt Foundation (Grant No. CHN/1127072).

\section{APPENDIX A: REACTION FORCE INDUCED TORQUE ON A SPHEROID ANALYZED BY GEOMETRICAL OPTICS}

As shown in Fig. 15, a focused Gaussian beam propagating in the $O_{\mathrm{P}^{-}} x z$ plane illuminates a spheroid with $z$ axis being its rotational symmetric axis. For simplicity in the 

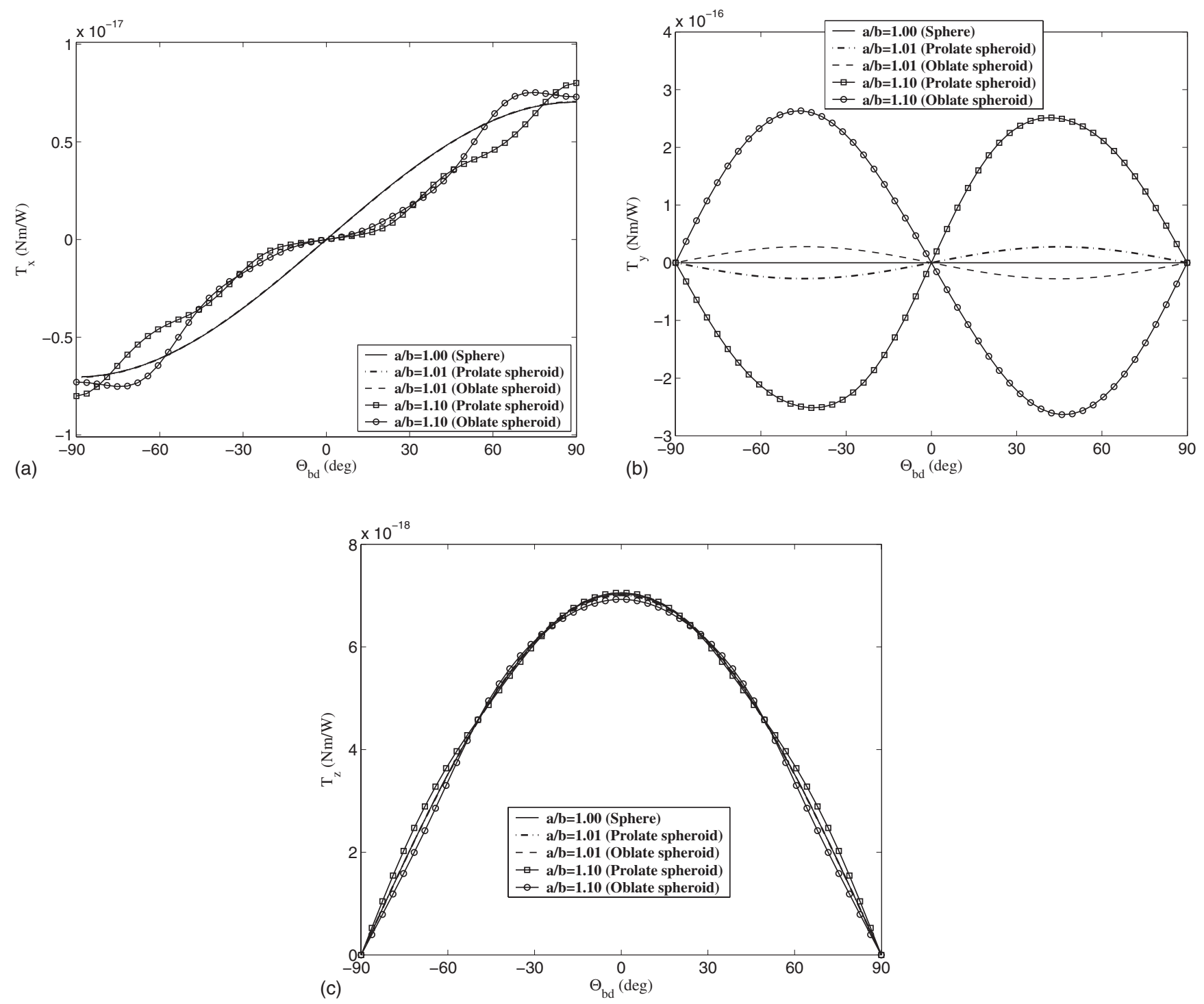

FIG. 13. Torque components $T_{\mathrm{x}}(\mathrm{a}), T_{\mathrm{y}}(\mathrm{b})$, and $T_{\mathrm{z}}(\mathrm{c})$ versus the incident angle $\left(\Theta_{\mathrm{bd}}\right)$ of the beam. The particles used here are same as those used for Fig. 3 (having a complex refractive index) while the beam is now right-circularly polarized. In (b), $T_{\mathrm{y}}$ is zero for a sphere at all incident angles $\left(T_{\mathrm{y}}=0\right)$. Note that in $(\mathrm{a})$, the $T_{\mathrm{x}}-\Theta_{\mathrm{bd}}$ curves of a sphere and the spheroids of axis ratio $a / b=1.01$ nearly coincide with each other.

qualitative analysis of the torque about the $y$ axis, this beam is approximated by the ray $A_{0} O_{0}$. The polarization of the electric field of the beam and the equivalent ray is in the $O_{\mathrm{P}^{-}} x z$ plane. Assuming the spheroid is very large compared to the width of the focused beam, the curvature of the spheroidal surface in the $y$ direction can be neglected and the prolate spheroid simplifies to an ellipse in the $O_{\mathrm{P}^{-}} x z$ plane. Keeping the incident ray fixed and rotating the ellipse $90^{\circ}$ about the $y$ axis gives the case for an oblate spheroid. The torque exerted on the spheroid is contributed by rays of all orders, namely,

$$
\boldsymbol{T}=\left(\boldsymbol{R}_{0} \times \boldsymbol{F}_{0}^{R}+\sum_{p=1}^{\infty} \boldsymbol{R}_{p} \times \boldsymbol{F}_{p}^{R}\right)+\left(\boldsymbol{R}_{0} \times \boldsymbol{F}_{0}^{T}+\sum_{p=1}^{\infty} \boldsymbol{R}_{p} \times \boldsymbol{F}_{p}^{T}\right),
$$

where $\boldsymbol{R}_{p}$ is the vector from the particle center $O_{\mathrm{P}}$ to the intersection point of the ray and the elliptical boundary and
$\boldsymbol{F}_{p}^{R}$ and $\boldsymbol{F}_{p}^{T}$ are the reaction forces exerted on the spheroid in response to the $p$ th order reflected and transmitted rays, respectively. The first terms in two brackets, $\boldsymbol{R}_{0} \times \boldsymbol{F}_{0}^{R}$ and $\boldsymbol{R}_{0}$ $\times \boldsymbol{F}_{0}^{T}$, represent the torques induced in response to the reflected and transmitted rays of zeroth order $(p=0)$. They occur when the incident ray hits the spheroid at an incident angle $\theta_{i, 0}$ and is refracted into spheroid via angle $\theta_{\mathrm{r}, 0}$. Higher orders $(p \geq 1)$ mean the rays experiencing $(p-1)$ internal reflections. The rate of momentum contained in a propagating light ray is related to the ray's power $P$ by

$$
\boldsymbol{M}=\frac{M_{m} P}{c} \boldsymbol{k}
$$

where $\boldsymbol{k}$ is the unit vector in the propagation direction of the ray, $c$ is light speed in the vacuum, and $M_{m}$ is the refractive index of the medium in which the ray propagates. The am- 

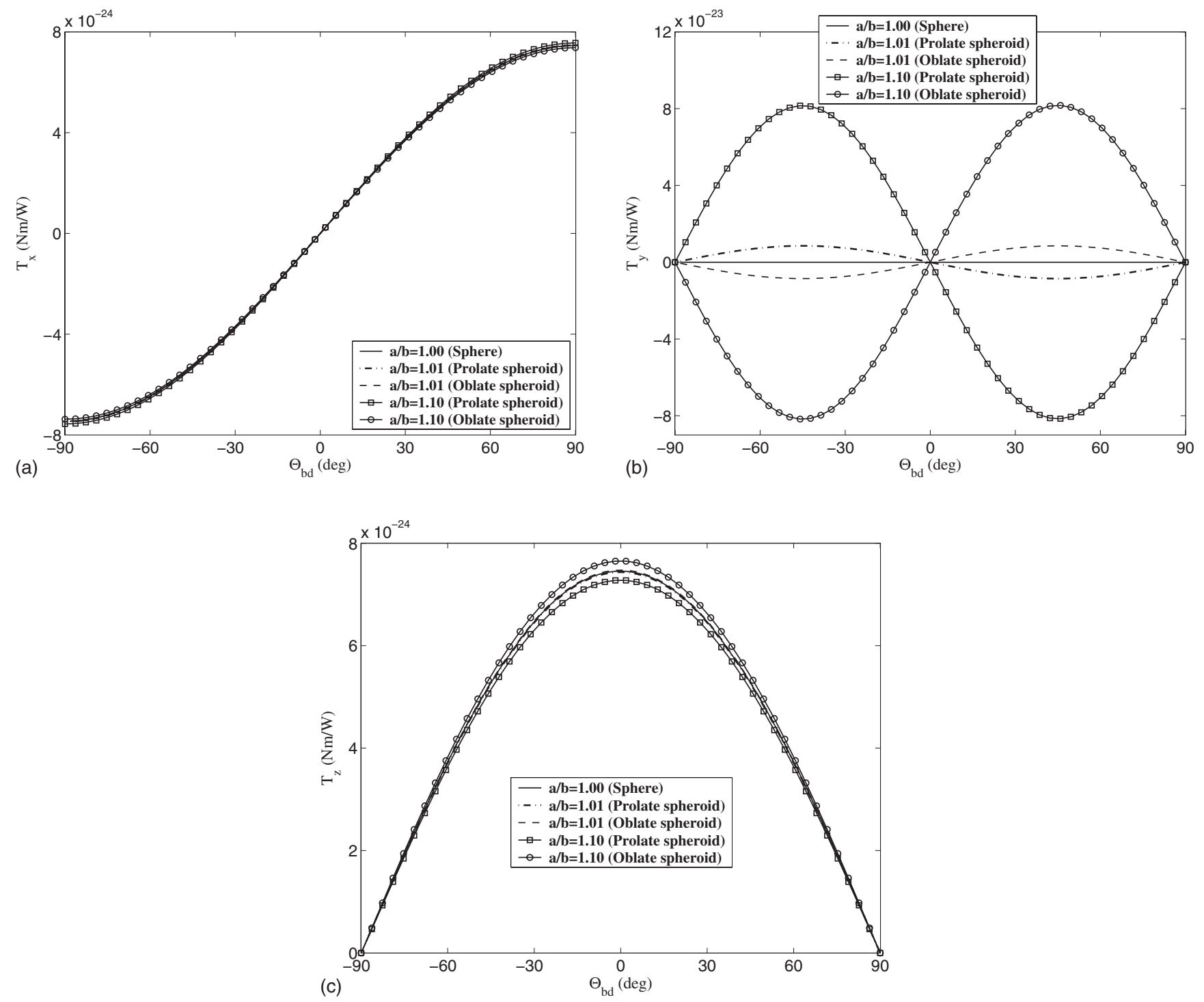

FIG. 14. Torque components $T_{\mathrm{x}}(\mathrm{a}), T_{\mathrm{y}}(\mathrm{b})$, and $T_{\mathrm{z}}$ (c) versus the incident angle $\left(\Theta_{\mathrm{bd}}\right)$ of the circularly polarized beam. The beam is same as that used for Fig. 13. The particles are also same as those used for Fig. 13, but their size is changed to $r_{\mathrm{s}}=10 \mathrm{~nm}$. Note that in (a), the $T_{\mathrm{x}}-\Theta_{\mathrm{bd}}$ curves of a sphere and the spheroids of axis ratios $a / b=1.01$ and 1.10 nearly coincide with each other. In (b), $T_{\mathrm{y}}$ is zero for a sphere at all incident angles $\left(T_{\mathrm{y}}=0\right)$.

plitude of the reaction force in response to a reflected ray of order $p$ can be evaluated by

$$
\begin{aligned}
F_{p}^{R}=\Delta M^{R}=2 \frac{M_{0}}{c} I_{p}^{R} \Delta A \sin \left(\frac{\pi}{2}-\theta_{i, p}\right), \\
\quad p=0, \quad 2 \frac{M_{r}^{\prime}}{c} I_{p}^{R} \Delta A \sin \left(\frac{\pi}{2}-\theta_{i, p}\right), \quad p \geq 1,
\end{aligned}
$$

where $\Delta A$ is the area illuminated by the ray. And the force in response to a transmitted ray is

$$
F_{p}^{T}=\Delta M^{T}=\frac{M_{0}}{c} I_{p}^{T} \Delta A\left[M_{r}^{2}+1-2 M_{r}^{2} \cos \left(\theta_{i, p}-\theta_{r, p}\right)\right]^{1 / 2} .
$$

In these equations $M_{0}$ and $M^{\prime}$ are the refractive indices of the medium and the particle, respectively, the relative value
$M=M^{\prime} / M_{0}=M_{\mathrm{r}}+i M_{\mathrm{i}}$, with the subscripts $r$ and $i$ indicating the real and imaginary parts of the complex respectively, and $I_{p}$ is the intensity of $p$ th order ray calculated by

$$
\begin{gathered}
I_{p}^{R}=\left|A_{p}^{R}\right|^{2}=\left|E_{0} \varepsilon_{p}^{R} \exp \left(-k_{\mathrm{I}} M_{i} L_{p}\right)\right|^{2}, \\
I_{p}^{T}=\left|A_{p}^{T}\right|^{2}=\left|E_{0} \varepsilon_{p}^{T} \exp \left(-k_{\mathrm{I}} M_{i} L_{p}\right)\right|^{2},
\end{gathered}
$$

where $A_{p}$ is the remaining amplitude after $p-1$ internal reflections, $E_{0}$ is the original amplitude of the incident ray, $k_{\mathrm{I}}$ is the wave number in medium, $L_{p}$ is the optical path experienced by an emergent ray of $p$ th order, and $\varepsilon_{p}$ are the factors indicating the amplitude of $p$ th order reflected or transmitted rays. They are evaluated by 


$$
\varepsilon_{p}^{R}=r_{p}, \quad p=0, \quad\left(1-r_{0}^{2}\right)^{1 / 2} \prod_{n=1}^{p} r_{n}, \quad p \geq 1,
$$

and

$$
\varepsilon_{p}^{T}=\left(1-r_{p}^{2}\right)^{1 / 2}, \quad p=0, \quad\left(1-r_{0}^{2}\right)^{1 / 2}\left(1-r_{p}^{2}\right)^{1 / 2} \prod_{n=1}^{p-1} r_{n}, \quad p \geq 1 .
$$

Note that the geometrical optics developed here deals with nonabsorbing or slightly absorbing particles. In the latter case, the absorption is taken into account through the addition of an exponential factor $\exp \left(-k_{I} M_{i} L_{p}\right)$ to the amplitude attenuation, as indicated in Eqs. (A5) and (A6). And the Fresnel reflection coefficient $r_{p}$ associated with the polarization parallel to the $O_{\mathrm{P}^{-}} x z$ plane is evaluated by using the real part of the complex refractive index, namely

$$
r_{p}= \begin{cases}\frac{M_{r} \cos \theta_{i}^{p}-\cos \theta_{r}^{p}}{M_{r} \cos \theta_{i}^{p}+\cos \theta_{r}^{p}}, & p=0, \\ \frac{\cos \theta_{i}^{p}-M_{r} \cos \theta_{r}^{p}}{\cos \theta_{i}^{p}+M_{r} \cos \theta_{r}^{p}}, & p \geq 1 .\end{cases}
$$

The direction of the reaction forces resulting from reflection and refraction can be obtained from the following unit vector operation:

$$
\begin{array}{r}
\boldsymbol{u}_{F_{p}^{R}}=\left(\boldsymbol{u}_{A_{p} \rightarrow O_{p}}-\boldsymbol{u}_{O_{p} \rightarrow B_{p}}\right) /\left|\boldsymbol{u}_{A_{p} \rightarrow O_{p}}-\boldsymbol{u}_{O_{p} \rightarrow B_{p}}\right|, \quad \text { (A10) } \\
\boldsymbol{u}_{F_{p}^{T}}=\left\{\begin{array}{l}
\left(\boldsymbol{u}_{A_{p} \rightarrow O_{p}}-M_{r} \boldsymbol{u}_{O_{p} \rightarrow C_{p}}\right) /\left|\boldsymbol{u}_{A_{p} \rightarrow O_{p}}-M_{r} \boldsymbol{u}_{O_{p} \rightarrow C_{p}}\right|, p=0, \\
\left(M_{r} \boldsymbol{u}_{A_{p} \rightarrow O_{p}}-\boldsymbol{u}_{O_{p} \rightarrow C_{p}}\right) /\left|M_{r} \boldsymbol{u}_{A_{p} \rightarrow O_{p}}-\boldsymbol{u}_{O_{p} \rightarrow C_{p}}\right|, p \geq 1 .
\end{array}\right.
\end{array}
$$

where $\boldsymbol{u}$ represents the unit vector along the direction indicated by its subscript.

Knowing the incident angle $\theta_{i}^{p}$, the refraction angle $\theta_{r}^{p}$, the deflection angle $\theta_{p}$ measured with respect to the positive $z$ axis for the emergent ray of $p$ th order and its intersection points with the boundary $\left(x_{p}, z_{p}\right)$ from a ray-tracing program [46], $\boldsymbol{u}_{F_{p}^{R}}$ and $\boldsymbol{u}_{F_{p}^{T}}$ have the following explicit form:

$$
\begin{aligned}
\boldsymbol{u}_{F_{p}^{R}}= & \frac{\left\{\cos \Theta_{\mathrm{bd}}-\cos \left[\Theta_{\mathrm{bd}}+q\left(\pi-2 \theta_{i}^{p}\right)\right]\right\} \boldsymbol{k}+\left\{\sin \Theta_{\mathrm{bd}}-\sin \left[\Theta_{\mathrm{bd}}+q\left(\pi-2 \theta_{i}^{p}\right)\right]\right\} \boldsymbol{i}}{\sqrt{2\left(1+\cos 2 \theta_{i}^{p}\right)}}, \quad p=0, \\
& \frac{\left\{\cos \left[\theta_{p}+q\left(\theta_{r}^{p}-\theta_{i}^{p}\right)\right]-\cos \left[\theta_{p}+q\left(\pi+\theta_{r}^{p}+\theta_{i}^{p}\right)\right]\right\} \boldsymbol{k}}{\sqrt{2\left[1-\cos 2\left(\pi-2 \theta_{i}^{p}\right)\right]}}+\frac{\left\{\sin \left[\theta_{p}+q\left(\theta_{r}^{p}-\theta_{i}^{p}\right)\right]-\sin \left[\theta_{p}+q\left(\pi+\theta_{r}^{p}+\theta_{i}^{p}\right)\right]\right\} \boldsymbol{i}}{\sqrt{2\left[1-\cos 2\left(\pi-2 \theta_{i}^{p}\right)\right]}}, \quad p \geq 1,
\end{aligned}
$$

and

$$
\begin{gathered}
\boldsymbol{u}_{F_{p}^{T}}=\frac{\left\{\cos \Theta_{\mathrm{bd}}-M_{r} \cos \left[\Theta_{\mathrm{bd}}-q\left(\theta_{i}^{p}-\theta_{r}^{p}\right)\right]\right\} \boldsymbol{k}+\left\{\sin \Theta_{\mathrm{bd}}-M_{r} \sin \left[\Theta_{\mathrm{bd}}-q\left(\theta_{i}^{p}-\theta_{r}^{p}\right)\right]\right\} \boldsymbol{i}}{\sqrt{M_{r}^{2}+1-2 M_{r} \cos \left(\theta_{i}^{p}-\theta_{r}^{p}\right)}}, \quad p=0, \\
\frac{\left\{M_{r} \cos \left[\theta_{p}+q\left(\theta_{r}^{p}-\theta_{i}^{p}\right)\right]-\cos \theta_{p}\right\} \boldsymbol{k}+\left\{M_{r} \sin \left[\theta_{p}+q\left(\theta_{r}^{p}-\theta_{i}^{p}\right)\right]-\sin \theta_{p}\right\} \boldsymbol{i}}{\sqrt{M_{r}^{2}+1-2 M_{r} \cos \left(\theta_{r}^{p}-\theta_{i}^{p}\right)}}, \quad p \geq 1,
\end{gathered}
$$

where $\boldsymbol{i}$ and $\boldsymbol{k}$ are the unit vectors along the positive $x$ and $z$ axes, respectively, and

$$
q=\operatorname{sgn}\left(\boldsymbol{u}_{A_{p} \rightarrow O_{p}} \times \boldsymbol{u}_{R_{p}}\right),
$$

where the unit radial vector $\boldsymbol{u}_{R_{p}}$ is determined from the intersection points of the emergent ray with the boundary, namely, $\boldsymbol{u}_{R_{p}}=z_{p} / \sqrt{z_{p}^{2}+x_{p}^{2}} \boldsymbol{k}+x_{p} / \sqrt{z_{p}^{2}+x_{p}^{2}} \boldsymbol{i}$.

The outward directed unit normal vector $\boldsymbol{n}$ to the surface of the spheroid can be evaluated by

$$
\boldsymbol{n}_{p}=\left\{\begin{array}{l}
\cos \left[\Theta_{\mathrm{bd}}+q\left(\pi-\theta_{i}^{p}\right)\right] \boldsymbol{k}+\sin \left[\Theta_{\mathrm{bd}}+q\left(\pi-\theta_{i}^{p}\right)\right] \boldsymbol{i}, p=0, \\
\cos \left(\theta_{p}+q \theta_{r}^{p}\right) \boldsymbol{k}+\sin \left(\theta_{p}+q \theta_{r}^{p}\right) \boldsymbol{i}, p \geq 1 .
\end{array}\right.
$$

Invoking the Snell's law of refraction, after several steps of algebraic operations it can be proved that

$$
\boldsymbol{n}_{p} \cdot \boldsymbol{u}_{F_{p}^{R}}=\left\{\begin{array}{l}
-1, \quad p=0, \\
1, \quad p \geq 1
\end{array}\right.
$$

and 


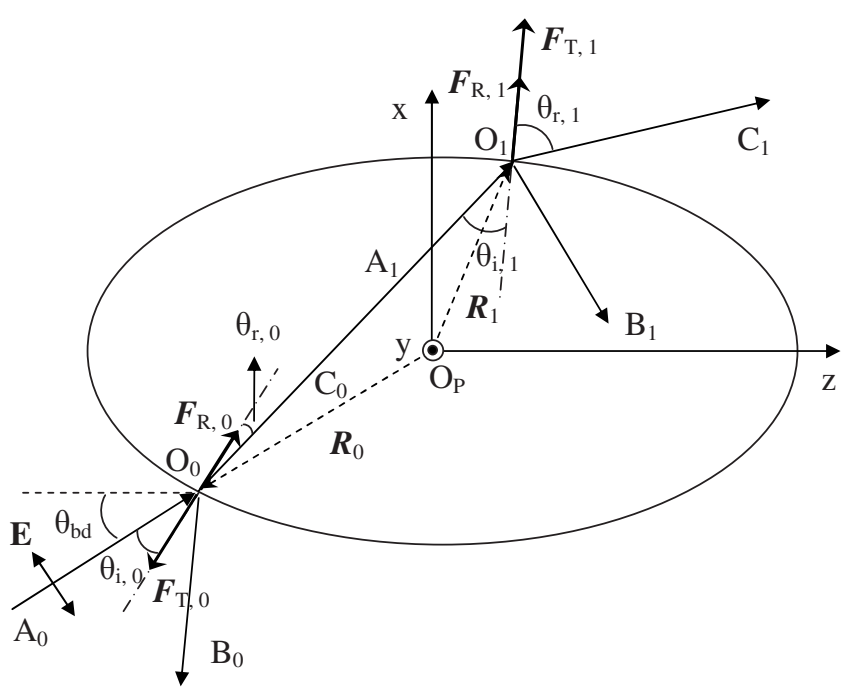

FIG. 15. Ray tracing in a prolate spheroid for a torque calculation.

$$
\boldsymbol{n}_{p} \cdot \boldsymbol{u}_{F_{p}^{T}}=1 .
$$

Equations (A16) and (A17) indicate that torque components caused by all reflection and refraction are along or opposite to the outwardly directed local normal vector to the boundary surface of the spheroid. As a special case, the normal vector to a spherical surface is always along the direction of the lever-arm $\boldsymbol{R}$ so that both $\boldsymbol{R}_{p} \times \boldsymbol{F}_{p}^{R}$ and $\boldsymbol{R}_{p} \times \boldsymbol{F}_{p}^{T}$ are equal to zero and no torque is produced.

Note that the geometrical optics model proposed here is used to analyze the torque contributed by reaction force. The orbit-absorption torque induced via photon absorption is not included.

\section{APPENDIX B: POLARIZATION INDUCED TORQUE ON A SPHEROID PREDICTED BY THE DIPOLE METHOD}

\section{Theory}

For the electromagnetic plane wave of incident angle $\Theta_{b d}$ and polarization angle $\Phi_{\mathrm{bd}}$ in the coordinates of the spheroid, the incident electric field can be written as

$$
\begin{gathered}
\mathbf{E}_{\mathrm{i}}(\boldsymbol{r}, t)=\frac{1}{2}\left[\mathbf{E}_{\mathrm{i}}(\boldsymbol{r}) \exp (-i \omega t)+\mathbf{E}_{\mathrm{i}}^{*}(\boldsymbol{r}) \exp (i \omega t)\right], \\
\mathbf{E}_{\mathrm{i}}(\boldsymbol{r})=E_{\mathrm{ix}} \mathbf{e}_{\mathrm{x}}+E_{\mathrm{iy}} \mathbf{e}_{\mathrm{y}}+E_{\mathrm{iz}} \mathbf{e}_{\mathrm{z}} \\
E_{\mathrm{ix}}=E_{0} \cos \Phi_{\mathrm{bd}} \sin \left(\frac{\pi}{2}+\Theta_{\mathrm{bd}}\right) \\
E_{\mathrm{iy}}=E_{0} \sin \Phi_{\mathrm{bd}} \\
E_{\mathrm{iz}}=E_{0} \cos \Phi_{\mathrm{bd}} \cos \left(\frac{\pi}{2}+\Theta_{\mathrm{bd}}\right)
\end{gathered}
$$

where $\boldsymbol{r}$ is the position vector. Then the induced electric dipole moment can be evaluated by

$$
\begin{gathered}
\mathbf{p}(\boldsymbol{r}, t)=\frac{1}{2}\left[\mathbf{p}_{\mathrm{i}}(\boldsymbol{r}) \exp (-i \omega t)+\mathbf{p}_{\mathrm{i}}^{*}(r) \exp (-i \omega t)\right], \\
\mathbf{p}_{\mathrm{i}}(\mathbf{r})=\stackrel{\leftrightarrow}{\alpha} \cdot \mathbf{E}_{\mathrm{i}}(\mathbf{r}),
\end{gathered}
$$

where $\stackrel{\leftrightarrow}{\alpha}$ where is the polarizability tensor, with the following matrix form for a spheroid:

$$
\overleftrightarrow{\alpha}=\left[\begin{array}{lll}
\alpha_{\perp} & 0 & 0 \\
0 & \alpha_{\perp} & 0 \\
0 & 0 & \alpha_{\|}
\end{array}\right],
$$

where $\alpha_{\|}$and $\alpha_{\perp}$ are the polarizability factors parallel and perpendicular to the rotational axis of the spheroid, respectively. In more detail, we have

$$
\mathbf{p}_{\mathrm{i}}(\boldsymbol{r})=\operatorname{Re}\left[\alpha_{\perp} \mathrm{E}_{\mathrm{ix}} \mathbf{e}_{\mathrm{x}}+\alpha_{\perp} \mathrm{E}_{\mathrm{iy}} \mathbf{e}_{\mathrm{y}}+\alpha_{\|} \mathrm{E}_{\mathrm{iz}} \mathbf{e}_{\mathrm{z}}\right] .
$$

The torque exerted by the electric field on such a dipole moment can be calculated by [31]

$$
\mathbf{T}=\mathbf{p} \times \mathbf{E} .
$$

Over a time period $\left[-t^{\prime}, t^{\prime}\right]$, we have the time-average torque as follows:

$$
\langle\mathbf{T}\rangle=\frac{\int_{-t^{\prime}}^{+t^{\prime}}(\mathbf{p} \times \mathbf{E}) d t}{2 t^{\prime}} .
$$

Namely,

$$
\begin{aligned}
\langle\mathbf{T}\rangle= & \frac{1}{4}\left[\mathbf{p}_{\mathrm{i}}(\boldsymbol{r}) \times \mathbf{E}_{\mathrm{i}}(\boldsymbol{r}) \exp (-2 i \omega t)+\mathbf{p}_{\mathrm{i}}(\boldsymbol{r}) \times \mathbf{E}_{\mathrm{i}}^{*}(\boldsymbol{r})+\mathbf{p}_{\mathrm{i}}^{*}(\boldsymbol{r})\right. \\
& \left.\times \mathbf{E}_{\mathrm{i}}(\boldsymbol{r})+\mathbf{p}_{\mathrm{i}}^{*}(\boldsymbol{r}) \times \mathbf{E}_{\mathrm{i}}^{*}(\boldsymbol{r}) \exp (2 i \omega t)\right]
\end{aligned}
$$

Letting $t^{\prime}$ be much larger than the optical cycle $T=2 \pi / \omega$, the contributions of the first and fourth terms can be neglected, so that Eq. (B6) becomes

$$
\langle\mathbf{T}\rangle=\frac{1}{4}\left[\mathbf{p}_{\mathrm{i}}(\boldsymbol{r}) \times \mathbf{E}_{\mathrm{i}}^{*}(\boldsymbol{r})+\mathbf{p}_{\mathrm{i}}^{*}(\boldsymbol{r}) \times \mathbf{E}_{\mathrm{i}}(\boldsymbol{r})\right] .
$$

Substituting Eq. (B1) and Eq. (B4) into Eq. (B8) yields

$$
\begin{aligned}
\langle\mathbf{T}\rangle= & \frac{1}{4} \mathrm{E}_{0}^{2} \operatorname{Re}\left(\alpha_{\|}-\alpha_{\perp}\right)\left[\sin \left(2 \Phi_{\mathrm{bd}}\right) \sin \left(\Theta_{\mathrm{bd}}\right) \mathbf{e}_{\mathrm{x}}\right. \\
& \left.-\sin \left(2 \Theta_{\mathrm{bd}}\right) \cos ^{2}\left(\Phi_{\mathrm{bd}}\right) \mathbf{e}_{\mathrm{y}}\right],
\end{aligned}
$$

where Re indicates the real part of the complex expression.

\section{Parameters}

Denoting the permittivities of the vacuum, the medium, and the spheroid by $\varepsilon_{0}, \varepsilon_{\mathrm{I}}$, and $\varepsilon_{\mathrm{II}}$, respectively, and the electric conductivity of the particle by $\sigma_{\mathrm{II}}$, the complex dielectric constant of the spheroid becomes $\bar{\varepsilon}_{\mathrm{II}}=\varepsilon_{\mathrm{II}}+\frac{4 \pi \sigma_{\mathrm{II}}}{{ }_{\mathrm{I}}} i$ and the complex relative refractive index is $M=\left(\bar{\varepsilon}_{\mathrm{II}} / \varepsilon_{\mathrm{I}}\right)^{1 / 2}$.

Then we have the following polarizability factors for a spheroid with volume $V$ :

$$
\alpha_{\|}=\frac{V \varepsilon_{\mathrm{I}}\left(M^{2}-1\right)}{1+\left(M^{2}-1\right) L_{\|}},
$$




$$
\alpha_{\perp}=\frac{V \varepsilon_{\mathrm{I}}\left(M^{2}-1\right)}{1+\left(M^{2}-1\right) L_{\perp}},
$$

where the depolarization factor $L$ can be evaluated by [47]

$$
\begin{aligned}
L_{\|} & =-\frac{\gamma^{-2}}{1-\gamma^{-2}}+\frac{\gamma^{-2}}{2\left(1-\gamma^{-2}\right)^{3 / 2}} \ln \frac{1+\left(1-\gamma^{-2}\right)^{1 / 2}}{1-\left(1-\gamma^{-2}\right)^{1 / 2}}, \\
L_{\perp} & =\frac{1}{2\left(1-\gamma^{-2}\right)}-\frac{\gamma^{-2}}{4\left(1-\gamma^{-2}\right)^{3 / 2}} \ln \frac{1+\left(1-\gamma^{-2}\right)^{1 / 2}}{1-\left(1-\gamma^{-2}\right)^{1 / 2}},
\end{aligned}
$$

for a prolate spheroid and

$$
\begin{aligned}
L_{\|}= & \frac{1}{1-\gamma^{-2}}-\frac{\pi \gamma^{-1}}{2\left(1-\gamma^{-2}\right)^{3 / 2}} \\
& +\frac{\gamma^{-1}}{\left(1-\gamma^{-2}\right)^{3 / 2}} \arctan \left(\frac{\gamma^{-2}}{1-\gamma^{-2}}\right)^{1 / 2}, \\
L_{\perp}= & -\frac{\gamma^{-2}}{2\left(1-\gamma^{-2}\right)}+\frac{\pi \gamma^{-1}}{4\left(1-\gamma^{-2}\right)^{3 / 2}} \\
& -\frac{\gamma^{-1}}{2\left(1-\gamma^{-2}\right)^{3 / 2}} \arctan \left(\frac{\gamma^{-2}}{1-\gamma^{-2}}\right)^{1 / 2},
\end{aligned}
$$

for an oblate one, where $\gamma$ is the axis ratio equal to $a / b$. The summation of the depolarization factors is unity, namely, $2 L_{\perp}+L_{\|}=1$. Note that for a prolate spheroid $L_{\|}<\frac{1}{3}$ and $L_{\|}$ $<L_{\perp}$, while for a oblate one $L_{\|}>\frac{1}{3}$ and $L_{\|}>L_{\perp}$. Extracting
$\varepsilon_{\mathrm{I}}$ from $\alpha_{\perp}$ and $\alpha_{\|}$and invoking the relation between the prefactor $\varepsilon_{\mathrm{I}} \mathrm{E}_{0}^{2}$ and the light speed in a medium of refractive index $M_{0}$ by $\varepsilon_{\mathrm{I}} \mathrm{E}_{0}^{2}=\frac{2 M_{0}}{c}$, Eq. (B9) can be reformed to

$$
\begin{aligned}
\langle\mathbf{T}\rangle= & \frac{M_{0}}{2 c} \operatorname{Re}\left\{\frac{\left(M^{2}-1\right)^{2}\left(L_{\perp}-L_{\|}\right) V}{\left[1+\left(M^{2}-1\right) L_{\|}\right]\left[1+\left(M^{2}-1\right) L_{\perp}\right]}\right\} \\
& \times\left[\sin \left(2 \Phi_{\mathrm{bd}}\right) \sin \left(\Theta_{\mathrm{bd}}\right) \mathbf{e}_{\mathrm{x}}-\sin \left(2 \Theta_{\mathrm{bd}}\right) \cos ^{2}\left(\Phi_{\mathrm{bd}}\right) \mathbf{e}_{\mathrm{y}}\right] .
\end{aligned}
$$

\section{Special cases}

When $\Phi_{\mathrm{bd}}=0^{\circ}$, Eq. (B13) becomes

$$
\begin{aligned}
\langle\mathbf{T}\rangle= & \frac{M_{0}}{2 c} \operatorname{Re}\left\{\frac{\left(M^{2}-1\right)^{2}\left(L_{\|}-L_{\perp}\right) V}{\left[1+\left(M^{2}-1\right) L_{\|}\right]\left[1+\left(M^{2}-1\right) L_{\perp}\right]}\right\} \\
& \times \sin \left(2 \Theta_{\mathrm{bd}}\right) \mathbf{e}_{\mathrm{y}} .
\end{aligned}
$$

Therefore the rotation is about the $y$ axis. The maximum of $T_{\mathrm{y}}$ occurs at $2\left|\Theta_{\mathrm{bd}}\right|=90^{\circ}$, namely, $\left|\Theta_{\mathrm{bd}}\right|=45^{\circ}$. There is no torque at $\Theta_{\mathrm{bd}}=0^{\circ}$ or $\pm 90^{\circ}$.

When $\Theta_{\mathrm{bd}}=90^{\circ}$, Eq. (B13) becomes

$$
\begin{aligned}
\langle\mathbf{T}\rangle= & \frac{M_{0}}{2 c} \operatorname{Re}\left\{\frac{\left(M^{2}-1\right)^{2}\left(L_{\perp}-L_{\|}\right) V}{\left[1+\left(M^{2}-1\right) L_{\|}\right]\left[1+\left(M^{2}-1\right) L_{\perp}\right]}\right\} \\
& \times \sin \left(2 \Phi_{\mathrm{bd}}\right) \mathbf{e}_{\mathrm{x}} .
\end{aligned}
$$

Therefore the rotation is about the $x$ axis. The maximum of $T_{\mathrm{x}}$ occurs at $2\left|\Phi_{\mathrm{bd}}\right|=90^{\circ}$, namely, $\left|\Phi_{\mathrm{bd}}\right|=45^{\circ}$. There is no torque at $\Phi_{\mathrm{bd}}=0^{\circ}$ or $\pm 90^{\circ}$.
[1] A. Ashkin, Phys. Rev. Lett. 24, 156 (1970).

[2] A. Ashkin, Science 210, 1081 (1980).

[3] A. Ashkin and J. M. Dziedzic, Science 235, 1517 (1987)

[4] A. Ashkin, J. M. Dziedzic, and T. Yamane, Nature (London) 330, 769 (1987).

[5] A. Ashkin and J. M. Dziedzic, Proc. Natl. Acad. Sci. U.S.A. 86, 7914 (1989).

[6] K. C. Neumann and S. M. Block, Rev. Sci. Instrum. 75, 2787 (2004).

[7] M. E. J. Friese, T. A. Nieminen, N. R. Heckenberg, and H. Rubinsztein-Dunlop, Nature (London) 394, 348 (1998).

[8] J. Guck, R. Ananthakrishnan, T. J. Moon, C. C. Cunningham, and J. Käs, Phys. Rev. Lett. 84, 5451 (2000).

[9] P. L. Marston and J. H. Crichton, Phys. Rev. A 30, 2508 (1984).

[10] P. Chylek, J. T. Kiehl, and M. K. W. Ko, Appl. Opt. 17, 3019 (1978).

[11] J. S. Kim and S. S. Lee, J. Opt. Soc. Am. 73, 303 (1983).

[12] S. Chang and S. S. Lee, J. Opt. Soc. Am. B 2, 1853 (1985).

[13] G. Gouesbet, B. Maheu, and G. Gréhan, J. Opt. Soc. Am. A 5, 1427 (1988).

[14] J. P. Barton, D. R. Alexander, and S. A. Schaub, J. Appl. Phys. 66, 4594 (1989).

[15] H. Polaert, G. Gréhan, and G. Gouesbet, Opt. Commun. 155, 169 (1998)
[16] V. V. Kotlyar and A. G. Nalimov, Opt. Express 14, 6316 (2006).

[17] T. A. Nieminen, H. Rubinsztein-Dunlop, and N. R. Heckenberg, J. Quant. Spectrosc. Radiat. Transf. 70, 627 (2001).

[18] S. H. Simpson and S. Hanna, J. Opt. Soc. Am. A 24, 430 (2007).

[19] D. C. Benito, S. H. Simpon, and S. Hanna, Opt. Express 16, 2942 (2008).

[20] R. C. Gauthier, J. Opt. Soc. Am. B 14, 3323 (1997).

[21] R. C. Gauthier, J. Opt. Soc. Am. B 14, 782 (1997).

[22] R. C. Gauthier, Appl. Phys. Lett. 67, 2269 (1995).

[23] R. C. Gauthier, Appl. Phys. Lett. 69, 2015 (1996).

[24] Y. Harada and T. Asakura, Opt. Commun. 124, 529 (1996).

[25] A. Rohrbach and E. H. K. Stelzer, J. Opt. Soc. Am. A 18, 839 (2001).

[26] G. Gouesbet and G. Gréhan, Atomization Sprays 10, 277 (2000).

[27] F. Xu, K. Ren, G. Gouesbet, G. Gréhan, and X. Cai, J. Opt. Soc. Am. A 24, 119 (2007).

[28] F. Xu, K. Ren, G. Gouesbet, X. Cai, and G. Gréhan, Phys. Rev. E 75, 026613 (2007).

[29] A. M. Stewart, Eur. J. Phys. 26, 635 (2005).

[30] J. D. Jackson, Classical Electrodynamics, 2nd ed. (Wiley, New York, 1975).

[31] J. A. Stratton, Electromagnetic Theory (McGraw-Hill, New 
York 1941).

[32] F. Xu, K. Ren, and X. Cai, J. Opt. Soc. Am. A 24, 109 (2007).

[33] C. Flammer, Spheroidal Wave Functions (Stanford University Press, Stanford, CA, 1957).

[34] J. P. Barton and D. R. Alexander, J. Appl. Phys. 66, 2800 (1989).

[35] X. Ma, J. Q. Lu, R. S. Brock, K. M. Jacobs, P. Yang, and X. H. Hu, Phys. Med. Biol. 48, 4165 (2003).

[36] E. Evans and Y. C. Fung, Microvasc. Res. 37, 7410 (1998).

[37] L. W. Davis, Phys. Rev. A 19, 1177 (1979).

[38] K. F. Ren, G. Gouesbet, and G. Gréhan, Appl. Opt. 37, 4218 (1998).

[39] G. Gouesbet, J. Opt. Soc. Am. A 16, 1641 (1999).

[40] J. A. Lock and G. Gouesbet, J. Opt. Soc. Am. A 11, 2503 (1994).
[41] G. Gouesbet and J. A. Lock, J. Opt. Soc. Am. A 11, 2516 (1994).

[42] G. Gouesbet, C. Letellier, K. F Ren, and G. Gréhan, Appl. Opt. 35, 1537 (1996).

[43] C. F. Bohren and D. Huffman, Absorption and Scattering of Light by Small Particles (Wiley, New York, 1983).

[44] K. C. Kao, Br. J. Appl. Phys. 12, 629 (1961).

[45] S. Bayoudh, T. A. Nieminen, N. R. Heckenberg, and H. Rubinsztein-Dunlop, J. Mod. Opt. 50, 1581 (2003).

[46] F. Xu, K. F. Ren, X. Cai, and J. Shen, Appl. Opt. 45, 5000 (2006).

[47] N. J. Rivette and J. C. Baygents, Chem. Eng. Sci. 51, 5205 (1996).

[48] P. C. Chaumet and C. Billaudeau, J. Appl. Phys. 101, 023106 (2007). 